\title{
Quantitative Untersuchung der Anhangsorgane der Haut bei dem Deutschen.")
}

\author{
Von
}

Kaneo Yamada.

Aus dem Anatomischen Institut der Keio Universität, Tokyo.

Inhalt.

I. Einleitung. . . . . . . . . . . . . . . . . . . . . . : . . 722

II. Material und Methode . . . . . . . . . . . . . . . . . . . 722

III. Eigene Befunde . . . . . . . . . . . . . . . . . . . . . . . . 725

1. Menge der Schweissdrüse . . . . . . . . . . . . . . . . 725

2. Menge der Talgdrüse . . . . . . . . . . . . . . . . . . . . . 730

3. Menge des Haarbalgmuskels . . . . . . . . . . . . . . . . . 736

IV. Tabellarische Zusammenstellung . . . . . . . . . . . . . . . . . . 744

1. Schweissdrüsse. . . . . . . . . . . . . . . . . . . . . 744

a. Menge und Ausmündungszahl der Drüse in den einzelnen Körperteilen.

b. Reihenfolge der Mengengrösse und Ausmündungszahl der Drüse in einzelnen Körperteilen.

c. Menge und Ausmündungszahl der Drüse in den Hauptkörperabschnitten.

2. Talgdrüse. . . . . . . . . . . . . . . . . . . . . . . 746

a. Menge der Drüse in den einzelnen Körperteilen.

b. Menge der Drüse in den Hauptkörperabschnitten.

3. Haarbalgmuskel. . . . . . . . . . . . . . . . . . . . . . . . 747

a. Menge des Muskels in den einzelnen Körperteilen.

b. Menge des Muskels in den Hauptkörperabschnitten.

V. Zusammenfassung. . . . . . . . . . . . . . . . . . . . 748

1) Diese Abhandlung ist die 4. Mitteilung von "Quantitative Untersuchungen der Anhangsorgane der Haut von K. Okajima." 


\section{Einleitung.}

Über die Bestimmung der Menge der Hautanhangsorgane von den verschiedenen Körperregionen in verschiedenen Rassen, Altersstufen und in beiden Geschlechtern haben schon Okajima ${ }^{1)}$ bei den Säugetieren, Taniguchi $i^{2}$ ) bei dem japanischen Neugeborenen und Koibuchi ${ }^{3)}$ bei dem japanischen Kind eingehende Untersuchungen gemacht. Nun werde ich im folgenden die Resultate, welche bei einem Deutschen gewonnen wurden, berichten.

\section{Material und Methode.}

Das mir zur Verfügung gestellte Material, das von Herm Prof. Stadtmüller des Anatomischen Institutes zu Göttingen Herrn Prof. Okajima überreicht wurde, stanmt aus einem 45 jährigen deutschen Selbstmörder (europäisches Rassengemisch, Mitteldeutschland), der sich erhängt hat. Die Leiche wurde nach dem Tode sogleich mit Alkohol-Formol-Glyzerin-Karbolsäure-Gemisch injiziert und kam dann in Alkohol. 2 Tage später wurden die Hautstücke mit genügendem Unterhautgewebe davon entnommen und in 10\% Formalinlösung gebracht, worin sie konserviert wurden. Die Hautstücke von ca. $1.2 \mathrm{qcm}$ Grösse wurden sorgfältig von diesem Material herausgeschnitten, in Zelloidin eingebettet und in Flächenschnittserien von $40 \mu$ oder $50 \mu$ Dicke zerlegt. Folgende 8 Körperteile wurden verfolgt.

I. Kopf :

1. Stirn

2. Hinterhaupt

II. Rumpf :

3. Bauch (linke Seite)

4. Rücken (rechte Seite)

III. Obere Extremität :

5. Oberarm (Streckseite der mittleren Höhe, rechts)

6. Vorderarm (Streckseite der mittleren Höhe, rechts)

1) Quantitative Untersuchung des Haarbalgmuskels bei den Säugetieren. Folia Anat. Japon. Bd. VII. 1929.

2) Quantitative Untersuchung der Anhangsorgane der Haut bei dem japanischen Nengeborenen. Folia Anat. Japon. Bd. IX. 1931.

3) Quantitative Untersuchung der Anhangsorgane der Haut bei dem japanischen Kind. Folia Anat. Japon. Bd. X. 1932. 
IV. Untere Extremität :

7. Oberschenkel (laterale Seite der oberen Höhe, links)

8. Unterschenkel (laterale Seite der oberen Höhe, links)

Die Färbung geschah mit Hämatoxylin (Hansen)-Eosin.

Das Haar, der Haarbalgmuskel, die Talgdrüse und Schweissdrüse in jedem Schnitt wurden alle 30 mal vergrössert auf ein Papierstück mit dem bestimmten Gewicht projiziert gezeichnet; damn wurden die gezeichneten Gegenstände aus allen Pupierstücken mit Schere herausgeschnitten und mittelst der Torsionswage genau gewogen.

Zur Berechnung des Volumens brauchte ich folgende Gleichung nach Okajima :

$$
\mathrm{V}=\frac{\mathrm{FD} \Sigma \mathrm{W}}{\mathrm{W}_{1} \mathrm{~m}^{2}}
$$

$\mathrm{V}=$ Volumen der Gegenstände,

$\mathrm{F}=$ Flächeninhalt des einzelnen Papierstückes,

$\mathrm{W}=$ Gewicht des einzelnen Papieístückes,

$\mathrm{W}=$ Gewicht des Gegenstandes in einem Schnitt,

$\mathrm{D}=$ Dicke des Schnittes,

$\mathrm{m}=$ Vergrösserung.

Nun werde ich im folgenden meine Daten tabellarisch angeben. 


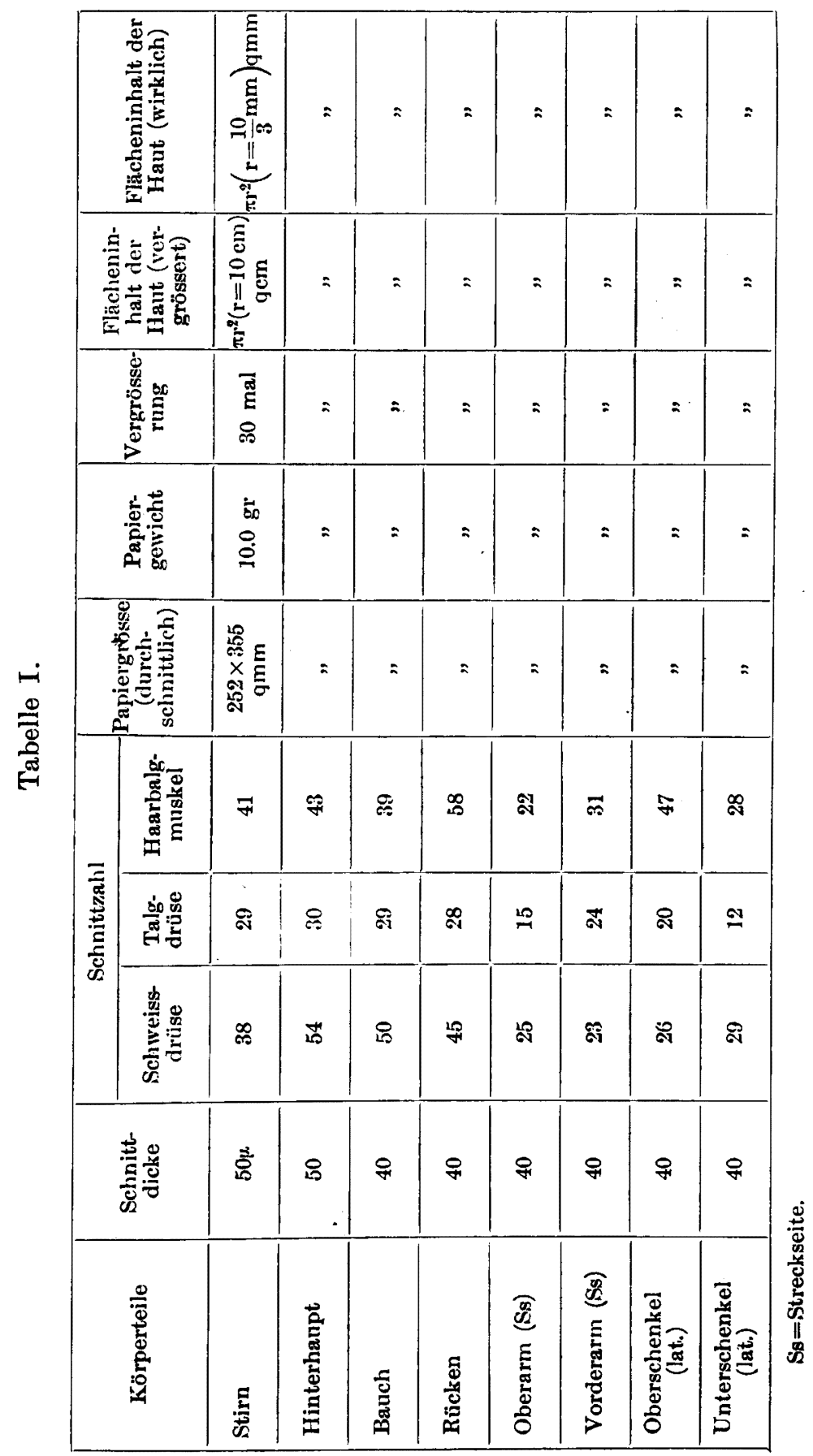




\section{Eigene Befunde.}

\section{A. Menge der Schweissdrüse.}

Tabelle 2. Schweissdrüse. Stirnhaut.

\begin{tabular}{|c|c|c|}
\hline $\begin{array}{l}\text { Schnitt- } \\
\text { nummer }\end{array}$ & $\begin{array}{l}\text { Zahl der } \\
\text { Drüsen- } \\
\text { stücke }\end{array}$ & $\begin{array}{c}\text { Gewicht der } \\
\text { ausgeschnit- } \\
\text { tenen Papier- } \\
\text { stücke }\end{array}$ \\
\hline $\begin{array}{r}1 \\
2 \\
3 \\
4 \\
5 \\
6 \\
7 \\
8 \\
9 \\
10 \\
11 \\
12 \\
13 \\
14 \\
15 \\
16 \\
17 \\
18 \\
19 \\
20 \\
21 \\
22 \\
23 \\
24 \\
25 \\
26 \\
27 \\
28 \\
29 \\
30 \\
31 \\
332 \\
33 \\
34 \\
35 \\
36 \\
37 \\
38\end{array}$ & $\begin{array}{r}2 \\
7 \\
22 \\
41 \\
36 \\
46 \\
50 \\
67 \\
75 \\
81 \\
90 \\
87 \\
95 \\
100 \\
111 \\
114 \\
108 \\
98 \\
97 \\
76 \\
68 \\
63 \\
67 \\
46 \\
35 \\
22 \\
15 \\
19 \\
12 \\
8 \\
3 \\
3 \\
4 \\
3 \\
4 \\
2 \\
1 \\
1\end{array}$ & $\begin{array}{c}1.3 \mathrm{mg} \\
19 \\
39.3 \\
95.7 \\
200.5 \\
277.5 \\
374 \\
453 \\
455 \\
514 \\
534 \\
595 \\
635.8 \\
601.8 \\
647 \\
594.3 \\
536 \\
484.8 \\
381.8 \\
326 \\
249.5 \\
201.2 \\
161 \\
141 \\
102 \\
78 \\
51.2 \\
41.8 \\
30 \\
19 \\
1.2 \\
11.2 \\
6.3 \\
4 \\
2.7 \\
2 \\
0.8 \\
0.8\end{array}$ \\
\hline & & 8880.3 \\
\hline
\end{tabular}

Volumen in $\pi \mathrm{r}^{2} \mathrm{gmm}\left(\mathrm{r}=\frac{10}{3} \mathrm{~mm}\right)=$

$\mathrm{V}\left(\right.$ in $\left.\pi \times\left(\frac{10}{3}\right)^{2} \mathrm{gmm}\right)=\frac{\mathrm{FDNIV}}{W_{1} \mathrm{~m}^{2}}$

$=\frac{(252 \times 355) \times 0.05 \times 8880.3}{10000 \times 30^{2}}$

$=\frac{39721581.9}{9000000}=4.4135091 \mathrm{cmm}$,

also in $1 \mathrm{qcm}$ umgerechnet

$\mathrm{V}($ in $1 \mathrm{gcm})=4.4135091 \times \frac{100}{\pi \times\left(\frac{10}{3}\right)^{2}}$

$=\frac{4.4135091 \times 100 \times 9}{\pi \times 100}=\frac{39.7215819}{\pi}$

$=12.6437 \mathrm{cmm}$.

Ferner in $1 \mathrm{qcm}$ gleichmässig verteilt angenommen, bildet die Drüse eine kontinuierliche Schicht in Ausgleichs(Durchschnitts-)dicke von

$\underline{12.6437 \mathrm{cmm}}$

$=0.126437 \mathrm{~mm}$ (rund 126.4u.). 
Tabelle 3. Schweissdrüse.

Hinterhaupthaut.

\begin{tabular}{|c|c|c|}
\hline $\begin{array}{l}\text { Schnitt- } \\
\text { nummer }\end{array}$ & $\begin{array}{c}\text { Zahl der } \\
\text { Drüsen- } \\
\text { stücke }\end{array}$ & $\begin{array}{l}\text { Gewicht der } \\
\text { ausgeschnit- } \\
\text { tenen Papier- } \\
\text { stücke }\end{array}$ \\
\hline $\begin{array}{r}1 \\
2 \\
3 \\
4 \\
5 \\
6 \\
7 \\
8 \\
9 \\
10 \\
11 \\
12 \\
13 \\
14 \\
15 \\
16 \\
17 \\
18 \\
19 \\
20 \\
21 \\
22 \\
23 \\
24 \\
25 \\
26 \\
27 \\
28 \\
29 \\
30 \\
31 \\
32 \\
33 \\
34 \\
35 \\
36 \\
37 \\
38 \\
39 \\
40 \\
41 \\
42 \\
43 \\
44 \\
45 \\
46 \\
47 \\
48 \\
49 \\
50 \\
51 \\
52 \\
53 \\
54\end{array}$ & $\begin{array}{r}2 \\
3 \\
3 \\
2 \\
2 \\
2 \\
2 \\
8 \\
14 \\
22 \\
24 \\
22 \\
25 \\
37 \\
52 \\
61 \\
69 \\
70 \\
73 \\
79 \\
82 \\
80 \\
84 \\
90 \\
75 \\
78 \\
58 \\
60 \\
56 \\
51 \\
56 \\
52 \\
49 \\
37 \\
37 \\
34 \\
37 \\
37 \\
33 \\
27 \\
27 \\
21 \\
20 \\
18 \\
19 \\
16 \\
14 \\
13 \\
8 \\
8 \\
4 \\
3 \\
2 \\
2 \\
1\end{array}$ & $\begin{array}{c}1 \mathrm{mg} \\
1.2 \\
2.5 \\
1.8 \\
1.5 \\
2 \\
7.5 \\
14 \\
23.3 \\
4.2 \\
61.5 \\
65 \\
91.8 \\
131 \\
141 \\
163 \\
198 \\
204 \\
215 \\
108.5 \\
230.2 \\
203 \\
194 \\
176 \\
177.5 \\
166.8 \\
146 \\
162.5 \\
140.6 \\
134.2 \\
127.8 \\
117.5 \\
125 \\
136.5 \\
121.5 \\
118.2 \\
99 \\
84 \\
80 \\
75 \\
71.3 \\
59 \\
46 \\
52.3 \\
38 \\
30.8 \\
23 \\
19 \\
16.5 \\
8.5 \\
4.5 \\
4 \\
3 \\
1 \\
\end{array}$ \\
\hline & & 4757.8 \\
\hline
\end{tabular}

$\mathrm{V}$ (in $\left.\pi \mathrm{r}^{\circ} \mathrm{qmm}\right)=2.3646266 \mathrm{cmm}$.

$\mathrm{V}$ (in $1 \mathrm{gcm})=6.7741 \mathrm{cmm}$.

Ausgleichsdicke $=0.067741 \mathrm{~mm}$

(rund 67.7 $\mu$ ). 
Quantitative Untersuchung der Anhangsorgane der Haut bei dem Deutschen.

Tabelle 4. Schweissdrüse. Bauchhaut.

\begin{tabular}{|c|c|c|}
\hline $\begin{array}{l}\text { Sclinitt- } \\
\text { nummer }\end{array}$ & $\begin{array}{l}\text { Zahl der } \\
\text { Drüsen- } \\
\text { stücke }\end{array}$ & $\begin{array}{l}\text { Gewicht der } \\
\text { ausgeschn. } \\
\text { Papierstücke }\end{array}$ \\
\hline 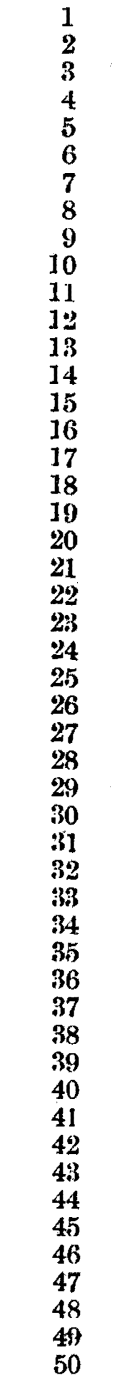 & 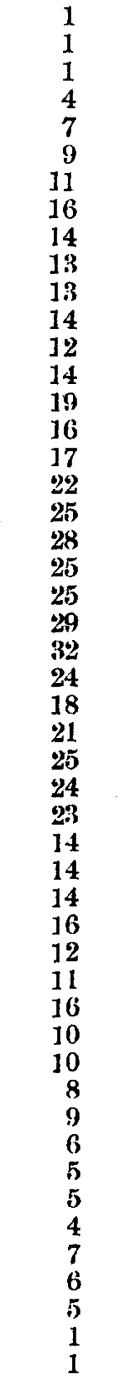 & 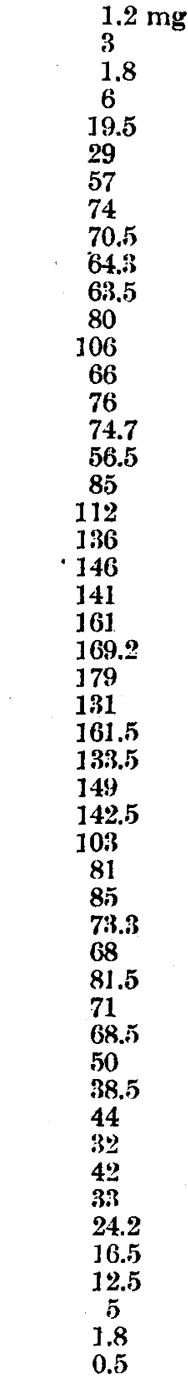 \\
\hline & & $36: 7$ \\
\hline
\end{tabular}

$\mathrm{V}$ (in $\left.\pi \mathrm{r}^{2} \mathrm{gmm}\right)=1.4420952 \mathrm{cmm}$. $V$ (in $1 \mathrm{qcm})=4.1313 \mathrm{cmm}$. Ausgleichsdicke $=0.041313 \mathrm{~mm}$
Tabelle 5. Schweissdrüse. Rückenhaut.

\begin{tabular}{|c|c|c|}
\hline $\begin{array}{l}\text { Schnitt- } \\
\text { nummer }\end{array}$ & $\begin{array}{l}\text { Zahl der } \\
\text { Drüsen- } \\
\text { stücke }\end{array}$ & $\begin{array}{l}\text { Gewicht der } \\
\text { ausgeschn. } \\
\text { Papierstücke }\end{array}$ \\
\hline $\begin{array}{r}1 \\
2 \\
3 \\
4 \\
5 \\
6 \\
7 \\
8 \\
9 \\
10 \\
11 \\
13 \\
13 \\
14 \\
15 \\
16 \\
17 \\
18 \\
19 \\
20 \\
21 \\
22 \\
23 \\
24 \\
25 \\
26 \\
27 \\
28 \\
29 \\
30 \\
31 \\
32 \\
33 \\
34 \\
35 \\
36 \\
37 \\
38 \\
39 \\
40 \\
41 \\
42 \\
43 \\
44 \\
45\end{array}$ & 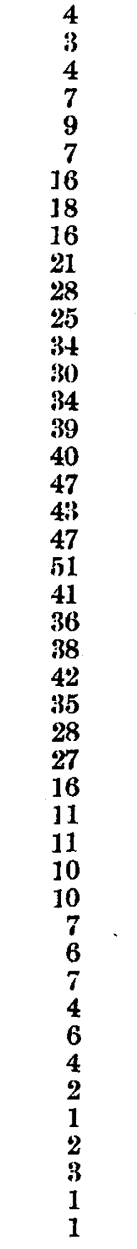 & $\begin{array}{l}5.8 \mathrm{mg} \\
17.5 \\
26.5 \\
37.5 \\
31.8 \\
30 \\
46.5 \\
58 \\
87 \\
99 \\
109.8 \\
133 \\
154 \\
161 \\
174 \\
199 \\
199.5 \\
211.3 \\
223.5 \\
226.5 \\
233.3 \\
220.7 \\
183.5 \\
170.5 \\
158 \\
130 \\
122.2 \\
125 \\
96 \\
70.7 \\
72 \\
43.5 \\
38 \\
20 \\
24 \\
21 \\
17 \\
15 \\
11 \\
9.3 \\
5 \\
4.5 \\
3 \\
0.5 \\
0.8\end{array}$ \\
\hline & & 4025.7 \\
\hline
\end{tabular}

$\mathrm{V}$ (in $\left.\pi \mathrm{r}^{2} \mathrm{qmm}\right)=1.60061832 \mathrm{cmm}$. $\mathrm{V}$ (in $1 \mathrm{qcm}$ ) $=4.5854 \mathrm{cmm}$.

Ausgleichsdicke $=0.045854 \mathrm{~mm}$

(rund 45.9 $\mu$ ).

(rund 41.3u) 
Tabelle 6. Schweissdrüse. Oberarm (Streckseite).

\begin{tabular}{|c|c|c|}
\hline $\begin{array}{l}\text { Schnitt- } \\
\text { nummer }\end{array}$ & $\begin{array}{l}\text { Zahl der } \\
\text { Drüsen- } \\
\text { stücke }\end{array}$ & $\begin{array}{l}\text { Gewicht der } \\
\text { ausgeschn. } \\
\text { Papierstücke }\end{array}$ \\
\hline 1 & 3 & $3 \mathrm{mg}$ \\
\hline 2 & 4 & 2.5 \\
\hline 3 & 9 & 9.5 \\
\hline 4 & 11 & 23 \\
\hline 5 & 19 & 40 \\
\hline 6 & 28 & 87 \\
\hline 7 & 21 & 112 \\
\hline 8 & 22 & 165.2 \\
\hline 9 & 30 & 152 \\
\hline 10 & 31 & 176 \\
\hline 11 & 28 & 216 \\
\hline 12 & 29 & 223 \\
\hline 13 & 29 & 224.5 \\
\hline 14 & 31 & 195 \\
\hline 15 & 26 & 210.8 \\
\hline 16 & 24 & 214 \\
\hline 17 & 25 & ] 73.5 \\
\hline 18 & 16 & 120.5 \\
\hline 19 & 17 & 82 \\
\hline 20 & 10 & 49 \\
\hline 21 & 7 & 37.5 \\
\hline 22 & 3 & 39 \\
\hline 23 & 3 & 37 \\
\hline 24 & 4 & 24.5 \\
\hline 25 & 2 & 10.5 \\
\hline & & 2627 \\
\hline
\end{tabular}

$\mathrm{V}$ (in $\left.\pi \mathrm{r}^{2} \mathrm{qmm}\right)=1.0444952 \mathrm{cmm}$.

$\mathrm{V}$ (in $1 \mathrm{qcm})=2.9923 \mathrm{cmm}$. Ausgleichsdicke $=0.029923 \mathrm{~mm}$
Tabelle 7. Schweissdrüse. Vorderarm (Streckseite).

\begin{tabular}{|c|c|c|}
\hline $\begin{array}{l}\text { Schnitt- } \\
\text { nummer }\end{array}$ & $\begin{array}{c}\text { Zahl der } \\
\text { Drüsen. } \\
\text { stücke }\end{array}$ & $\begin{array}{l}\text { Gewicht der } \\
\text { ausgesehn. } \\
\text { Papierstücke }\end{array}$ \\
\hline 1 & 2 & $6 \mathrm{mg}$ \\
\hline 2 & 3 & 14.5 \\
\hline 3 & 5 & 42.6 \\
\hline 4 & 14 & 80 \\
\hline 5 & 17 & -92.2 \\
\hline 6 & 25 & 148.5 \\
\hline 7 & 26 & 230.5 \\
\hline 8 & 25 & 320 \\
\hline 9 & 23 & 352.2 \\
\hline 10 & 31 & 335.5 \\
\hline 11 & 34 & 303.3 \\
\hline 12 & 34 & 287.5 \\
\hline 13 & 28 & 215 \\
\hline 14 & 21 & 204 \\
\hline 15 & 22 & 209.5 \\
\hline 16 & 19 & 191.8 \\
\hline 17 & 15 & 160.5 \\
\hline 18 & 15 & 103 \\
\hline 19 & 7 & 73 \\
\hline 20 & 7 & 69 \\
\hline 21 & 9 & 52.2 \\
\hline 22 & 5 & 22.3 \\
\hline 23 & 5 & 9 \\
\hline & & 3522.1 \\
\hline
\end{tabular}

$\mathrm{V}$ (in $\left.\pi \mathrm{r}^{2} \mathrm{gmm}\right)=1.40038696 \mathrm{cmm}$. $\mathrm{V}$ (in $1 \mathrm{gcm})=4.0118 \mathrm{cmm}$. Ausgleichsdicke $=0.040118 \mathrm{~mm}$

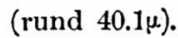

(rund 29.9u). 
Tabelle 8. Schweissdrüse. Oberschenkel (lateral).

\begin{tabular}{|c|c|c|}
\hline $\begin{array}{l}\text { Schnitt- } \\
\text { nummer }\end{array}$ & $\begin{array}{l}\text { Zahl der } \\
\text { Drüsen- } \\
\text { stūcke }\end{array}$ & $\begin{array}{l}\text { Gewicht der } \\
\text { ausgeschn. } \\
\text { Papierstūcke }\end{array}$ \\
\hline 1 & 3 & $1.5 \mathrm{mg}$ \\
\hline 2 & 3 & \\
\hline 3 & 7 & 7.5 \\
\hline 4 & 8 & 20 \\
\hline 5 & 8 & 31.3 \\
\hline 6 & 13 & 49 \\
\hline 7 & 18 & 79 \\
\hline 8 & 13 & 124.3 \\
\hline 9 & 17 & 118 \\
\hline 10 & 26 & 101 \\
\hline 11 & 25 & 109.5 \\
\hline 12 & 29 & 120 \\
\hline 13 & 19 & 128 \\
\hline 14 & 25 & 121 \\
\hline 15 & 36 & 127 \\
\hline 16 & 32 & 150.5 \\
\hline 17 & 29 & 165 \\
\hline 18 & 41 & 176 \\
\hline 19 & $\mathbf{3 0}$ & 131.5 \\
\hline 20 & 28 & 162 \\
\hline 21 & 28 & 139 \\
\hline 22 & 26 & 138.5 \\
\hline 23 & 31 & 169 \\
\hline 24 & 32 & 158.5 \\
\hline 25 & 22 & 147.3 \\
\hline 26 & 21 & 139.5 \\
\hline 27 & 24 & 138 \\
\hline 28 & 3 & 161 \\
\hline 29 & 22 & 151.3 \\
\hline 30 & 37 & 80.2 \\
\hline 31 & 13 & 58.5 \\
\hline 32 & 7 & 49 \\
\hline 33 & $\dot{5}$ & 27.5 \\
\hline 34 & 4 & 17.3 \\
\hline 35 & 1 & 8.3 \\
\hline 36 & 1 & 0.5 \\
\hline & & 3507.5 \\
\hline
\end{tabular}

$V\left(\right.$ in $\left.\pi \mathrm{r}^{2} \mathrm{gmm}\right)=1.394582 \mathrm{cmm}$.

$V$ (in $1 \mathrm{qcm})=3.9952 \mathrm{cmm}$.

Ausgleichsdicke $=0.039952 \mathrm{~mm}$

(rund $40 \%$
Tabelle 9. Schweissdrüse. Unterschenkel (lateral).

\begin{tabular}{|c|c|c|}
\hline $\begin{array}{l}\text { Schnitt- } \\
\text { nummer }\end{array}$ & $\begin{array}{l}\text { Zahl der } \\
\text { Drüsen- } \\
\text { stücke }\end{array}$ & $\begin{array}{l}\text { Gewicht der } \\
\text { ausgeschn. } \\
\text { Papierstūcke }\end{array}$ \\
\hline $\begin{array}{r}1 \\
2 \\
3 \\
4 \\
5 \\
6 \\
7 \\
8 \\
9 \\
10 \\
11 \\
12 \\
13 \\
14 \\
15 \\
16 \\
17 \\
18 \\
19 \\
20 \\
21 \\
22 \\
23 \\
24 \\
25 \\
26 \\
27 \\
28 \\
29\end{array}$ & $\begin{array}{r}2 \\
9 \\
13 \\
9 \\
17 \\
17 \\
22 \\
18 \\
21 \\
30 \\
45 \\
42 \\
42 \\
53 \\
48 \\
34 \\
32 \\
28 \\
29 \\
26 \\
19 \\
19 \\
14 \\
8 \\
6 \\
6 \\
1 \\
1 \\
1\end{array}$ & $\begin{array}{c}2.2 \mathrm{mg} \\
13.3 \\
41 \\
56 \\
112.3 \\
113.5 \\
124 \\
182.6 \\
202 \\
224 \\
286 \\
347 \\
403.8 \\
355.3 \\
386 \\
319 \\
291.8 \\
250 \\
238 \\
221 \\
175 \\
143 \\
90 \\
43 \\
28.2 \\
15.2 \\
9 \\
5.3 \\
1\end{array}$ \\
\hline & & 4678.7 \\
\hline
\end{tabular}

$V$ (in $\left.\pi r^{\circ} \mathrm{qmm}\right)=1.86025112 \mathrm{cmm}$. $V$ (in $1 \mathrm{qcm})=5.3292 \mathrm{cmm}$. Ausgleichsdicke $=0.053292 \mathrm{~mm}$

(rund 53.3u). 


\section{B. Menge der Talgdrüse.}

Tabelle 10. Talgdrüse.

Stiruhaut.

\begin{tabular}{|c|c|c|}
\hline $\begin{array}{l}\text { Schnitt- } \\
\text { nummer }\end{array}$ & $\begin{array}{l}\text { Zahl der } \\
\text { Drüsen- } \\
\text { stücke }\end{array}$ & $\begin{array}{l}\text { Gewicht der } \\
\text { ausgeschn. } \\
\text { Papierstücke }\end{array}$ \\
\hline $\begin{array}{r}1 \\
2 \\
3 \\
4 \\
5 \\
6 \\
7 \\
8 \\
9 \\
10 \\
11 \\
12 \\
13 \\
14 \\
15 \\
16 \\
17 \\
18 \\
19 \\
20 \\
21 \\
22 \\
23 \\
24 \\
25 \\
26 \\
27 \\
28 \\
29\end{array}$ & $\begin{array}{r}1 \\
4 \\
5 \\
20 \\
41 \\
54 \\
91 \\
112 \\
154 \\
170 \\
204 \\
233 \\
256 \\
262 \\
257 \\
218 \\
192 \\
177 \\
135 \\
110 \\
89 \\
75 \\
54 \\
40 \\
26 \\
10 \\
9 \\
6 \\
3\end{array}$ & $\begin{array}{c}0.3 \mathrm{mg} \\
4 \\
7.2 \\
32 . .3 \\
67.5 \\
94 \\
160 \\
215 \\
282 \\
357.2 \\
452 \\
493 \\
501.3 \\
527.5 \\
598 \\
478.8 \\
485 \\
426.8 \\
357 \\
274 \\
219 \\
164.5 \\
131.7 \\
8.3 \\
49 \\
26.8 \\
20 \\
12.2 \\
6.5\end{array}$ \\
\hline & & 6525.6 \\
\hline
\end{tabular}

$\mathrm{V}$ (in $\left.\pi \mathrm{r}^{2} \mathrm{qmm}\right)=3.2432232 \mathrm{cmm}$.

$\mathrm{V}$ (in $1 \mathrm{qcm}$ ) $=9.2911 \mathrm{cmm}$,

Ausgleichsdicke $=0.092911 \mathrm{~mm}$

(rund 92.9u).
Tabelle 11. Talgdrüse.

Hinterhaupthaut.

\begin{tabular}{|c|c|c|}
\hline $\begin{array}{l}\text { Schnitt- } \\
\text { nummer }\end{array}$ & $\begin{array}{l}\text { Zahl der } \\
\text { Drüsen- } \\
\text { stücke }\end{array}$ & $\begin{array}{l}\text { Gewicht der } \\
\text { ausgeschn. } \\
\text { Papierstücke }\end{array}$ \\
\hline $\begin{array}{l}1 \\
2 \\
3 \\
4 \\
5 \\
6 \\
7 \\
8 \\
9 \\
10 \\
11 \\
12 \\
13 \\
14 \\
15 \\
16 \\
17 \\
18 \\
19 \\
20 \\
21 \\
22 \\
23 \\
24 \\
25 \\
26 \\
27 \\
28 \\
29 \\
30\end{array}$ & $\begin{array}{r}1 \\
1 \\
5 \\
5 \\
17 \\
31 \\
38 \\
51 \\
110 \\
131 \\
175 \\
181 \\
216 \\
241 \\
248 \\
231 \\
218 \\
217 \\
191 \\
167 \\
152 \\
106 \\
107 \\
64 \\
41 \\
27 \\
16 \\
7 \\
6 \\
1\end{array}$ & $\begin{array}{c}1.5 \mathrm{mg} \\
2.2 \\
5.3 \\
8.3 \\
23.2 \\
48 \\
68 \\
98.2 \\
218 \\
308 \\
405.5 \\
489 \\
535.5 \\
546 \\
506.5 \\
514.5 \\
494.5 \\
442 \\
390.2 \\
364 \\
298 \\
227 \\
217.5 \\
115.5 \\
75 \\
45.5 \\
27 \\
13.7 \\
8 \\
1\end{array}$ \\
\hline & & 6496.6 \\
\hline
\end{tabular}

$\mathrm{V}$ (in $\left.\pi \mathrm{r}^{2} \mathrm{qmm}\right)=3.2288102 \mathrm{cmm}$.

$\mathrm{V}$ (in $1 \mathrm{gcm})=9.2498 \mathrm{cmm}$.

Ausgleichsdicke $=0.092498 \mathrm{~mm}$

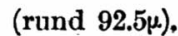


Tabelle 12. Talgdrüse. Bauchhaut.

\begin{tabular}{|c|c|c|}
\hline $\begin{array}{l}\text { Schnitt- } \\
\text { nummer }\end{array}$ & $\begin{array}{l}\text { Zahl der } \\
\text { Drüsen- } \\
\text { stücke }\end{array}$ & $\begin{array}{l}\text { Gewicht der } \\
\text { ausgeschn. } \\
\text { Papierstücke }\end{array}$ \\
\hline $\begin{array}{r}1 \\
2 \\
3 \\
4 \\
5 \\
6 \\
7 \\
8 \\
9 \\
10 \\
11 \\
12 \\
13 \\
14 \\
15 \\
16 \\
17 \\
18 \\
19 \\
20 \\
21 \\
22 \\
23 \\
24 \\
25 \\
26 \\
27 \\
28 \\
29\end{array}$ & $\begin{array}{l}2 \\
2 \\
3 \\
2 \\
2 \\
3 \\
4 \\
3 \\
3 \\
2 \\
2 \\
1 \\
1 \\
1 \\
1 \\
2 \\
3 \\
3 \\
3 \\
2 \\
2 \\
3 \\
2 \\
3 \\
5 \\
2 \\
1 \\
1 \\
1\end{array}$ & $\begin{array}{c}3 \mathrm{mg} \\
2.8 \\
6.5 \\
6.5 \\
9 \\
10.6 \\
9.3 \\
6 \\
3.3 \\
1.3 \\
1 \\
1 \\
1.5 \\
3.3 \\
7 \\
8 \\
7.5 \\
8.2 \\
6.8 \\
6.8 \\
5 \\
3.2 \\
3 \\
5 \\
6 \\
4.5 \\
2.5 \\
1 \\
1\end{array}$ \\
\hline & & 140.6 \\
\hline
\end{tabular}

$\mathrm{V}\left(\right.$ in $\left.\pi \mathrm{r}^{2} \mathrm{qmm}\right)=0.05590256 \mathrm{cmm}$.

$\mathrm{V}$ (in $1 \mathrm{qcm})=0.16015 \mathrm{cmm}$.

Ausgleichsdicke $=0.0016015 \mathrm{~mm}$

(rund 1.6p.).
Tabelle 13. Talgdrüse. Rückenhaut.

\begin{tabular}{|c|c|c|}
\hline $\begin{array}{l}\text { Schnitt- } \\
\text { nummer }\end{array}$ & $\begin{array}{l}\text { Zahl der } \\
\text { Drüsen- } \\
\text { stücke }\end{array}$ & $\begin{array}{l}\text { Gewicht der } \\
\text { ausgeschn. } \\
\text { Papierstücke }\end{array}$ \\
\hline $\begin{array}{r}1 \\
2 \\
3 \\
4 \\
5 \\
6 \\
7 \\
8 \\
9 \\
10 \\
11 \\
12 \\
13 \\
14 \\
15 \\
16 \\
17 \\
18 \\
19 \\
20 \\
21 \\
22 \\
23 \\
24 \\
25 \\
26 \\
27 \\
28\end{array}$ & $\begin{array}{r}1 \\
1 \\
1 \\
1 \\
1 \\
1 \\
1 \\
1 \\
3 \\
7 \\
7 \\
8 \\
8 \\
8 \\
7 \\
10 \\
10 \\
10 \\
11 \\
11 \\
7 \\
5 \\
4 \\
2 \\
2 \\
1 \\
1 \\
1\end{array}$ & $\begin{array}{l}0.2 \mathrm{mg} \\
0.5 \\
1 \\
1.5 \\
1 \\
1.7 \\
2.8 \\
3 \\
6.3 \\
11.3 \\
12 \\
15 \\
18 \\
19.5 \\
25 \\
33 \\
38 \\
39 \\
33.5 \\
31.2 \\
31 \\
26 \\
19.5 \\
14.8 \\
12.2 \\
4.8 \\
4.8 \\
1\end{array}$ \\
\hline & & 407.6 \\
\hline
\end{tabular}

$\mathrm{V}$ (in $\left.\pi \mathrm{r}^{2} \mathrm{qmm}\right)=0.16206176 \mathrm{cmm}$.

$\mathrm{V}$ (in $1 \mathrm{qcm})=0.4643 \mathrm{cmm}$.

Ausgleichsdicke $=0.004643 \mathrm{~mm}$

(rund 4.6u). 
Tabelle 14. Talgdrüse. Oberarm (Streckseite).

\begin{tabular}{|c|c|c|}
\hline $\begin{array}{c}\text { Schnitt- } \\
\text { nummer }\end{array}$ & $\begin{array}{c}\text { Zahl der } \\
\text { Drüsen- } \\
\text { stücke }\end{array}$ & $\begin{array}{c}\text { Gewicht der } \\
\text { ansgeschn. } \\
\text { Papierstücke }\end{array}$ \\
\cline { 1 - 1 } 1 & 1 & $0.5 \mathrm{mg}$ \\
2 & 2 & 1.5 \\
3 & 4 & 4.5 \\
4 & 6 & 9 \\
5 & 7 & 9 \\
6 & 6 & 12 \\
7 & 5 & 13 \\
8 & 6 & 16.5 \\
9 & 4 & 13 \\
10 & 2 & 11 \\
11 & 1 & 12 \\
12 & 2 & 14 \\
13 & 2 & 13.5 \\
14 & 2 & 7.5 \\
15 & 1 & 148 \\
\hline
\end{tabular}

$\mathrm{V}$ (in $\left.\pi \mathrm{r}^{2} \mathrm{qmm}\right)=0.0588448 \mathrm{cmm}$.

$V$ (in $1 \mathrm{qcm})=0.1686 \mathrm{cmm}$.

Ausgleichsdicke $=0.001686 \mathrm{~mm}$ (rund $1.7 \mu)$.

Tabelle 15. Talgdrüse.

Vorderarm (Streckseite).

\begin{tabular}{|c|c|c|}
\hline $\begin{array}{c}\text { Schnitt. } \\
\text { nummer }\end{array}$ & $\begin{array}{c}\text { Zahl der } \\
\text { Drüsen- } \\
\text { stïcke }\end{array}$ & $\begin{array}{c}\text { Gewicht der } \\
\text { ausgeschn. } \\
\text { Papierstücke }\end{array}$ \\
\cline { 1 - 2 } 1 & 1 & $0.3 \mathrm{mg}$ \\
2 & 1 & 0.3 \\
3 & 2 & 1 \\
4 & 3 & 1.7 \\
5 & 1 & 1.5 \\
6 & 3 & 4 \\
7 & 4 & 6.2 \\
8 & 4 & 9.2 \\
9 & 6 & 20.5 \\
10 & 10 & 29.5 \\
11 & 12 & 28 \\
12 & 9 & 22.8 \\
13 & 10 & 24 \\
14 & 10 & 27 \\
15 & 8 & 29 \\
16 & 4 & 27.3 \\
17 & 3 & 23.7 \\
18 & 5 & 4 \\
19 & 1 & 7 \\
20 & 1 & 10 \\
21 & 1 & 10.5 \\
22 & 2 & 4.5 \\
23 & 1 & 2 \\
24 & 1 & 316 \\
\hline & & \\
\hline
\end{tabular}

$V\left(\right.$ in $\left.\pi \mathrm{r}^{2} \mathrm{gmm}\right)=0.1256416 \mathrm{cmm}$.

$V$ (in $1 \mathrm{qcm}$ ) $=0.3599 \mathrm{cmm}$.

Ausgleichsdicke $=0.003599 \mathrm{~mm}$ (rund 3.6 $\mu$ ).
Tabelle 16. Talgdrüse.

Oberschenkel (lateral).

\begin{tabular}{|c|c|c|}
\hline $\begin{array}{c}\text { Schnitt- } \\
\text { nummer }\end{array}$ & $\begin{array}{c}\text { Zahl der } \\
\text { Drüsen- } \\
\text { stücke }\end{array}$ & $\begin{array}{c}\text { Gewicht der } \\
\text { ausgeschn. } \\
\text { Papierstïcke }\end{array}$ \\
\hline 1 & 1 & $0.5 \mathrm{mg}$ \\
2 & 3 & 4 \\
3 & 3 & 4.3 \\
4 & 4 & 4 \\
5 & 3 & 2.7 \\
6 & 2 & 2 \\
7 & 1 & 1.5 \\
8 & 1 & 1 \\
9 & 1 & 0.2 \\
10 & 1 & 0.5 \\
11 & 1 & 0.5 \\
12 & 1 & 5 \\
13 & 3 & 6.5 \\
14 & 4 & 10 \\
15 & 5 & 10.8 \\
16 & 5 & 8 \\
17 & 4 & 6.2 \\
18 & 2 & 0.8 \\
\hline 19 & 2 & 77.5 \\
20 & 1 & \\
\hline & & \\
\hline
\end{tabular}

$V$ (in $\left.\pi \mathrm{r}^{2} \mathrm{gmm}\right)=0.030814 \mathrm{cmm}$.

$V$ (in $1 \mathrm{gcm}$ ) $=0.08828 \mathrm{cmm}$.

Ausgleichsdicke $=0.0008828 \mathrm{~mm}$

(rund $0.9 \mu$ ).

Tabelle 17. Talgdrüse.

Unterschenkel (lateral).

\begin{tabular}{|c|c|c|}
\hline $\begin{array}{c}\text { Schnitt- } \\
\text { nummer }\end{array}$ & $\begin{array}{c}\text { Zahl der } \\
\text { Drüsen- } \\
\text { stücke }\end{array}$ & $\begin{array}{c}\text { Gewicht der } \\
\text { ausgeschn. } \\
\text { Papierstücke }\end{array}$ \\
\cline { 1 - 2 } 1 & 1 & $0.5 \mathrm{mg}$ \\
2 & 1 & 1 \\
3 & 4 & 2 \\
4 & 5 & 5 \\
5 & 3 & 5.3 \\
6 & 4 & 6.7 \\
7 & 2 & 2.5 \\
8 & 1 & 0.7 \\
9 & 1 & 3.5 \\
10 & 2 & 5 \\
11 & 3 & 2.5 \\
12 & 1 & 2.5 \\
\hline
\end{tabular}

$\mathrm{V}$ (in $\left.\mathrm{xr}^{2} \mathrm{qmm}\right)=0.01479072 \mathrm{cmm}$.

$\mathrm{V}$ (in $1 \mathrm{gcm})=0.0424 \mathrm{cmm}$.

Ausgleichsdicke $=0.000424 \mathrm{~mm}$ (rund $0.4 \mu$ ). 
Tabelle 18.

Volumen der Talgdrüsenindividuen in Stirnhaut.

\begin{tabular}{|c|c|c|c|c|c|}
\hline $\begin{array}{l}\text { Drüsen- } \\
\text { nummer }\end{array}$ & $\begin{array}{c}\text { Haarzahl } \\
\text { in einer } \\
\text { Gruppe }\end{array}$ & Schnittzahl & $\begin{array}{l}\text { Zahl der } \\
\text { Drüsen- } \\
\text { stücke }\end{array}$ & $\begin{array}{l}\text { Gewicht der } \\
\text { ausgeschn. } \\
\text { Papierstücke }\end{array}$ & $\begin{array}{l}\text { Volumen des } \\
\text { Drüsenindiv. }\end{array}$ \\
\hline $\begin{array}{l}\mathbf{a} \\
\mathbf{b} \\
\mathbf{c} \\
\mathrm{d} \\
\mathrm{e} \\
\mathbf{f} \\
\mathrm{g} \\
\mathrm{h} \\
\mathbf{i} \\
\mathbf{j}\end{array}$ & $\begin{array}{l}6 \\
6 \\
6 \\
6 \\
4 \\
4 \\
4 \\
3 \\
3 \\
3 \\
3\end{array}$ & $\begin{array}{l}13 \\
15 \\
18 \\
17 \\
15 \\
10 \\
18 \\
11 \\
10 \\
13\end{array}$ & $\begin{array}{l}65 \\
97 \\
84 \\
92 \\
67 \\
27 \\
75 \\
31 \\
20 \\
33\end{array}$ & $\begin{array}{l}156 \mathrm{mg} \\
232.5 \\
205 \\
145.5 \\
171.5 \\
32.5 \\
171 \\
70 \\
49 \\
80\end{array}$ & $\begin{array}{l}0.0775320 \mathrm{cmm} \\
0.1155525 \\
0.1018850 \\
0.0723135 \\
0.0852355 \\
0.0161525 \\
0.0849870 \\
0.0347900 \\
0.0243530 \\
0.0397600\end{array}$ \\
\hline \multicolumn{5}{|c|}{ durchschnittlich } & 0.0652561 \\
\hline
\end{tabular}

Tabelle 19.

Volumen der Talgdrüsenindividuen in Hinterbaupthaut.

\begin{tabular}{|c|c|c|c|c|c|}
\hline $\begin{array}{c}\text { Drüsen- } \\
\text { nummer }\end{array}$ & $\begin{array}{c}\text { Haarzahl } \\
\text { in einer } \\
\text { Gruppe }\end{array}$ & Schnittzahl & $\begin{array}{c}\text { Zahl der } \\
\text { Drüsen- } \\
\text { stücke }\end{array}$ & $\begin{array}{c}\text { Gewicht der } \\
\text { ausgeschn. } \\
\text { Papierstücke }\end{array}$ & $\begin{array}{c}\text { Volumen des } \\
\text { Drüsenindiv. }\end{array}$ \\
\hline a & 6 & 18 & 97 & $206 \mathrm{mg}$ & $0.1023820 \mathrm{cmm}$ \\
b & 6 & 15 & 86 & 142.5 & 0.0708225 \\
c & 5 & 18 & 72 & 138 & 0.0685860 \\
d & 4 & 17 & 71 & 182 & 0.0904540 \\
e & 4 & 17 & 54 & 131 & 0.0651070 \\
f & 3 & 20 & 54 & 156.5 & 0.0777805 \\
g & 3 & 13 & 37 & 62.2 & 0.0309134 \\
h & 3 & 19 & 68 & 209 & 0.1038730 \\
i & 3 & 17 & 59 & 116 & 0.0576520 \\
j & 3 & 15 & 35 & 68 & 0.0337960 \\
\hline \multicolumn{2}{|r|}{ durchschnittlich } & & & 0.0701366 \\
\hline
\end{tabular}

Tabelle 20.

Volumen der Talgdrüsenindividuen in Bauchhaut.

\begin{tabular}{|c|c|c|c|c|c|}
\hline $\begin{array}{l}\text { Drüsen- } \\
\text { nummer }\end{array}$ & $\begin{array}{c}\text { Haarzahl } \\
\text { in einer } \\
\text { Gruppe }\end{array}$ & Schnittzahl & $\begin{array}{l}\text { Zahl der. } \\
\text { Drüsen. } \\
\text { stücke }\end{array}$ & $\begin{array}{l}\text { Gewicht der } \\
\text { ausgeschn. } \\
\text { Papierstücke }\end{array}$ & $\begin{array}{l}\text { Volumen des } \\
\text { Drüsenindiv. }\end{array}$ \\
\hline $\begin{array}{l}\mathbf{a} \\
\mathbf{b} \\
\mathbf{c} \\
\mathbf{d} \\
\mathbf{e} \\
\mathbf{f} \\
\mathbf{g} \\
\mathbf{h} \\
\mathbf{i} \\
\mathbf{j}\end{array}$ & $\begin{array}{l}3 \\
2 \\
2 \\
2 \\
2 \\
2 \\
2 \\
2 \\
2 \\
2 \\
2\end{array}$ & $\begin{array}{l}4 \\
4 \\
7 \\
9 \\
3 \\
3 \\
8 \\
6 \\
6 \\
8\end{array}$ & $\begin{array}{r}6 \\
4 \\
10 \\
12 \\
3 \\
3 \\
8 \\
6 \\
7 \\
8\end{array}$ & $\begin{array}{l}5.5 \mathrm{mg} \\
4 \\
37 \\
21 \\
2 \\
2 \\
30.5 \\
13 \\
13.3 \\
19\end{array}$ & $\begin{array}{l}0.0021868 \mathrm{cmm} \\
0.0015904 \\
0.0147112 \\
0.0083496 \\
0.0007952 \\
0.0007952 \\
0.0121268 \\
0.0051688 \\
0.0052881 \\
0.0075544\end{array}$ \\
\hline \multicolumn{5}{|c|}{ durchschnittlich } & 0.0058566 \\
\hline
\end{tabular}


Tabelle 21.

Volumen der Talgdrüsenindividuen in Rückenhaut.

\begin{tabular}{|c|c|c|c|c|c|}
\hline $\begin{array}{c}\text { Drüsen- } \\
\text { nummer }\end{array}$ & $\begin{array}{c}\text { Haarzahl } \\
\text { in einer } \\
\text { Gruppe }\end{array}$ & Schnittzahl & $\begin{array}{c}\text { Zahl der } \\
\text { Drüsen- } \\
\text { stücke }\end{array}$ & $\begin{array}{c}\text { Gewicht der } \\
\text { ausgeschn. } \\
\text { Papierstücke }\end{array}$ & $\begin{array}{c}\text { Volumen des } \\
\text { Drüsenindiv. }\end{array}$ \\
\hline a & 4 & 7 & 12 & $19.2 \mathrm{mg}$ & $0.0076339 \mathrm{cmm}$ \\
$\mathrm{b}$ & $\mathbf{3}$ & 11 & 19 & 52.5 & 0.0208740 \\
$\mathrm{c}$ & 3 & 12 & 18 & 104 & 0.0413504 \\
$\mathrm{~d}$ & 3 & 14 & 20 & 44 & 0.0174944 \\
$\mathrm{e}$ & 2 & 11 & 12 & 35.5 & 0.0141148 \\
$\mathrm{f}$ & 2 & 12 & 12 & 41 & 0.0163016 \\
$\mathrm{~g}$ & 2 & 8 & 8 & 22.5 & 0.0089460 \\
$\mathrm{~h}$ & 2 & 11 & 17 & 58.3 & 0.0231801 \\
i & 2 & 14 & 14 & 28.5 & 0.0113316 \\
$\mathrm{j}$ & 2 & 13 & 23 & 69 & 0.0274344 \\
\hline \multicolumn{2}{|l}{ durchschnittlich } & & & 0.0188661 \\
\hline
\end{tabular}

Tabelle 22.

Volụmen der Talgdrüsenindividuen in Oberarm (Streckseite).

\begin{tabular}{|c|c|c|c|c|c|}
\hline $\begin{array}{l}\text { Drüsen- } \\
\text { nummer }\end{array}$ & $\begin{array}{c}\text { Haarzahl } \\
\text { in einer } \\
\text { Gruppe }\end{array}$ & Schnittzahl & $\begin{array}{l}\text { Zahl der } \\
\text { Drüseu- } \\
\text { stücke }\end{array}$ & $\begin{array}{l}\text { Gewicht der } \\
\text { ausgeschn. } \\
\text { Papierstücke }\end{array}$ & $\begin{array}{l}\text { Volumen des } \\
\text { Drüsenindiv. }\end{array}$ \\
\hline $\begin{array}{c}\mathbf{a} \\
\mathbf{b} \\
\mathbf{c} \\
\mathbf{d} \\
\mathbf{e} \\
\mathbf{f} \\
\mathrm{g} \\
\mathbf{h} \\
\mathbf{i} \\
\mathbf{j}\end{array}$ & $\begin{array}{l}3 \\
3 \\
3 \\
3 \\
3 \\
3 \\
2 \\
2 \\
2 \\
2 \\
2\end{array}$ & $\begin{array}{r}5 \\
5 \\
6 \\
3 \\
5 \\
5 \\
3 \\
2 \\
11\end{array}$ & $\begin{array}{r}7 \\
7 \\
6 \\
3 \\
5 \\
5 \\
3 \\
2 \\
18\end{array}$ & $\begin{array}{l}15 \mathrm{mg} \\
11 \\
26 \\
-9 \\
9 \\
23.8 \\
8 \\
2.5 \\
2 \\
88\end{array}$ & $\begin{array}{l}0.0059640 \mathrm{cmm} \\
0.0043736 \\
0.0103376 \\
- \\
0.0035784 \\
0.0094629 \\
0.0031808 \\
0.0009940 \\
0.0007952 \\
0.0349888\end{array}$ \\
\hline \multicolumn{5}{|c|}{ durchschnittlich } & 0.0073675 \\
\hline
\end{tabular}

Tabelle 23.

Volumen der Talgdrüsenindividuen in Vorderarm (Streckseite).

\begin{tabular}{|c|c|c|c|c|c|}
\hline $\begin{array}{c}\text { Drüsen- } \\
\text { nummer }\end{array}$ & $\begin{array}{c}\text { Haarzahl } \\
\text { in einer } \\
\text { Gruppe }\end{array}$ & Schnittzahl & $\begin{array}{c}\text { Zahl der } \\
\text { Drüsen- } \\
\text { stücke }\end{array}$ & $\begin{array}{c}\text { Gewicht der } \\
\text { ausgeschn. } \\
\text { Papierstücke }\end{array}$ & $\begin{array}{c}\text { Volumen des } \\
\text { Drüsenindiv. }\end{array}$ \\
\hline a & 4 & 10 & 15 & $28 \mathrm{mg}$ & $0.0111328 \mathrm{cmm}$ \\
b & 3 & 11 & 19 & 125 & 0.0497000 \\
c & 3 & 10 & 14 & 31.5 & 0.0125244 \\
d & 3 & 9 & 13 & 72 & 0.0286272 \\
e & 3 & 9 & 10 & 22 & 0.0087472 \\
f & 2 & 6 & 8 & 9.5 & 0.0037772 \\
g & 2 & 7 & 14 & 25 & 0.0099400 \\
h & 2 & 8 & 9 & 26.2 & 0.0104171 \\
i & 2 & 10 & 11 & 44 & 0.0174944 \\
i j & 2 & 6 & 11 & 35 & 0.0139160 \\
\hline \multicolumn{2}{|l}{ durchschnittlich } & & & & 0.0166276 \\
\hline
\end{tabular}


Tabelle 24.

Volumen der Talgdrüsenindividuen in Oberschenkel (lateral).

\begin{tabular}{|c|c|c|c|c|c|}
\hline $\begin{array}{l}\text { Drüsen- } \\
\text { nummer }\end{array}$ & $\begin{array}{l}\text { Haarzahl } \\
\text { in einer } \\
\text { Gruppe }\end{array}$ & Schnittzahl & $\begin{array}{l}\text { Zahl der } \\
\text { Drüsen- } \\
\text { stücke }\end{array}$ & $\begin{array}{c}\text { Gewicht der } \\
\text { ausgeschn. } \\
\text { Papierstücke }\end{array}$ & $\begin{array}{l}\text { Volumen des } \\
\text { Drüsenindiv. }\end{array}$ \\
\hline $\begin{array}{l}\mathrm{a} \\
\mathrm{b} \\
\mathbf{c} \\
\mathrm{d} \\
\mathbf{e} \\
\mathbf{f} \\
\mathrm{g} \\
\mathrm{h} \\
\mathbf{i} \\
\mathbf{j}\end{array}$ & $\begin{array}{l}3 \\
3 \\
3 \\
2 \\
2 \\
2 \\
2 \\
2 \\
2 \\
2 \\
2 \\
1\end{array}$ & $\begin{array}{r}13 \\
10 \\
8 \\
6 \\
7 \\
5 \\
9 \\
4 \\
- \\
-\end{array}$ & $\begin{array}{r}13 \\
10 \\
8 \\
10 \\
8 \\
6 \\
13 \\
4 \\
-\end{array}$ & $\begin{array}{l}15.5 \mathrm{mg} \\
10 \\
13 \\
25 \\
11 \\
9 \\
25 \\
4.5 \\
-\end{array}$ & $\begin{array}{l}0.0061628 \mathrm{cmm} \\
0.0039760 \\
0.0051688 \\
0.0099400 \\
0.0043736 \\
0.0035784 \\
0.0099400 \\
0.0017892 \\
\text { - }\end{array}$ \\
\hline \multicolumn{5}{|c|}{ durchschnittlich } & 0.0044929 \\
\hline
\end{tabular}

Tabelle 25.

Volumen der Talgdrüsenindividuen in Unterschenkel (lateral).

\begin{tabular}{|c|c|c|c|c|c|}
\hline $\begin{array}{l}\text { Drüsen- } \\
\text { nummer }\end{array}$ & $\begin{array}{c}\text { Haarzahl } \\
\text { in einer } \\
\text { Gruppe }\end{array}$ & Schnittzahl & $\begin{array}{l}\text { Zahl der } \\
\text { Drüsen- } \\
\text { stücke }\end{array}$ & $\begin{array}{l}\text { Gewicht der } \\
\text { ausgeschn. } \\
\text { Papierstücke }\end{array}$ & $\begin{array}{l}\text { Volumen des } \\
\text { Drüsenindiv. }\end{array}$ \\
\hline $\begin{array}{l}\mathbf{a} \\
\mathbf{b} \\
\mathbf{c} \\
\mathrm{d} \\
\mathrm{e} \\
\mathrm{f} \\
\mathrm{g} \\
\mathrm{h} \\
\mathrm{i} \\
\mathrm{j}\end{array}$ & $\begin{array}{l}3 \\
3 \\
3 \\
2 \\
2 \\
2 \\
2 \\
2 \\
2 \\
2\end{array}$ & $\begin{array}{r}4 \\
4 \\
\quad \mathbf{3} \\
\mathbf{3} \\
\mathbf{2} \\
4 \\
4 \\
5 \\
5\end{array}$ & $\begin{array}{l}9 \\
7 \\
3 \\
6 \\
2 \\
4 \\
5 \\
6 \\
5\end{array}$ & $\begin{array}{l}8 \mathrm{mg} \\
6.5 \\
-1 \\
10 \\
2 \\
7 \\
6.5 \\
5.8 \\
12.5\end{array}$ & $\begin{array}{l}0.0031808 \mathrm{cmm} \\
0.0025844 \\
- \\
0.0003976 \\
0.0039760 \\
0.0007952 \\
0.0027832 \\
0,0025844 \\
0.0023061 \\
0.0049700\end{array}$ \\
\hline \multicolumn{5}{|c|}{ durchschnittlich } & 0.0023578 \\
\hline
\end{tabular}




\section{Menge des Haarbalgmuskels.}

Tabelle 26.

Muskel. Stimhaut.

\begin{tabular}{|c|c|c|}
\hline $\begin{array}{l}\text { Schnitt- } \\
\text { nummer }\end{array}$ & $\begin{array}{l}\text { Zahl der } \\
\text { Mustel- } \\
\text { stāeke }\end{array}$ & $\begin{array}{l}\text { Gevicht dex } \\
\text { ansgeschn. } \\
\text { Papienstūcke }\end{array}$ \\
\hline $\begin{array}{r}1 \\
2 \\
3 \\
4 \\
5 \\
6 \\
7 \\
8 \\
9 \\
10 \\
11 \\
12 \\
13 \\
14 \\
15 \\
16 \\
17 \\
18 \\
19 \\
20 \\
21 \\
99 \\
23 \\
24 \\
25 \\
36 \\
\mathbf{9 1} \\
28 \\
99 \\
30 \\
31 \\
32 \\
33 \\
34 \\
35 \\
36 \\
37 \\
38 \\
39 \\
40 \\
41\end{array}$ & $\begin{array}{r}1 \\
\mathbf{2} \\
4 \\
11 \\
\mathbf{2 1} \\
36 \\
41 \\
66 \\
80 \\
103 \\
111 \\
106 \\
110 \\
114 \\
107 \\
95 \\
92 \\
87 \\
85 \\
87 \\
78 \\
78 \\
60 \\
65 \\
48 \\
\mathbf{5 1} \\
38 \\
\mathbf{3 4} \\
\mathbf{9 3} \\
\mathbf{2 7} \\
\mathbf{3 0} \\
\mathbf{1 6} \\
9 \\
5 \\
\mathbf{2} \\
\mathbf{9} \\
1 \\
1 \\
1 \\
1 \\
1\end{array}$ & 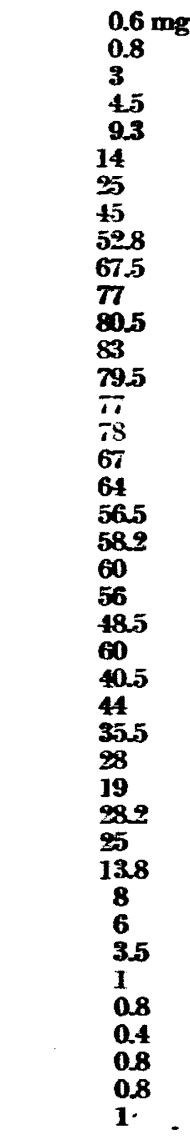 \\
\hline & & 1494 \\
\hline
\end{tabular}

$V$ (in $\left.x^{2} \mathrm{qmm}\right)=0.707728 \mathrm{cmm}$.

$V$ (in $1 \mathrm{gcm}$ ) $=20275 \mathrm{cmm}$.

Ausgleichsdicke $=0.030275 \mathrm{~mm}$

(rund 20.3 R)
Tabelle 27.

Muskel. Hinterhaupthaut.

\begin{tabular}{|c|c|c|}
\hline $\begin{array}{l}\text { Sehnitt- } \\
\text { nummer }\end{array}$ & $\begin{array}{l}\text { Zahl der } \\
\text { Inaskel } \\
\text { stücke }\end{array}$ & $\begin{array}{l}\text { Gewieht der } \\
\text { anseschn. } \\
\text { Papierstōcke }\end{array}$ \\
\hline \begin{tabular}{|c|}
1 \\
3 \\
4 \\
5 \\
6 \\
7 \\
8 \\
9 \\
10 \\
11 \\
12 \\
13 \\
14 \\
15 \\
16 \\
17 \\
18 \\
19 \\
90 \\
21 \\
29 \\
93 \\
94 \\
95 \\
96 \\
27 \\
38 \\
29 \\
30 \\
31 \\
32 \\
33 \\
34 \\
35 \\
36 \\
37 \\
38 \\
39 \\
40 \\
41 \\
49 \\
43 \\
3
\end{tabular} & $\begin{array}{r}1 \\
9 \\
3 \\
6 \\
9 \\
11 \\
15 \\
29 \\
31 \\
45 \\
53 \\
66 \\
78 \\
55 \\
94 \\
100 \\
95 \\
100 \\
107 \\
98 \\
92 \\
87 \\
92 \\
89 \\
86 \\
57 \\
89 \\
87 \\
81 \\
81 \\
80 \\
76 \\
68 \\
69 \\
69 \\
41 \\
39 \\
24 \\
15 \\
11 \\
6 \\
4 \\
1\end{array}$ & 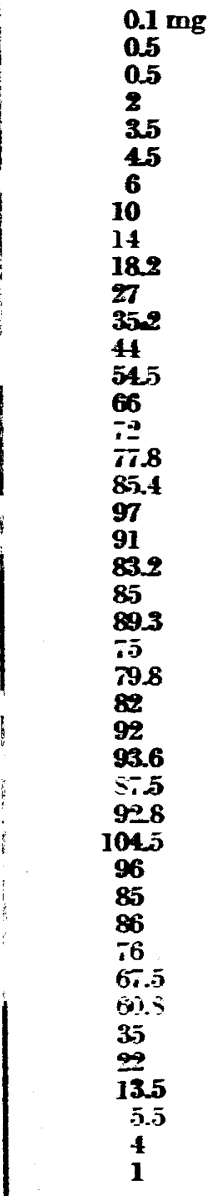 \\
\hline & & $\infty 65$ \\
\hline $\begin{array}{l}\text { in } 1 \\
\text { Ansgleich }\end{array}$ & $\begin{array}{l}\text { m) }=1.1 \\
=3170\end{array}$ & $\begin{array}{l}5 \mathrm{cmm} \\
\mathrm{n} . \\
\mathrm{mm}\end{array}$ \\
\hline
\end{tabular}

(rund 31.7 p) 
Tabelle 28.

Muskel. Bauchhaut.

\begin{tabular}{|c|c|c|}
\hline $\begin{array}{l}\text { Schnitt- } \\
\text { nummer }\end{array}$ & $\begin{array}{l}\text { Zahl der } \\
\text { Muskel- } \\
\text { stücke }\end{array}$ & $\begin{array}{l}\text { Gewicht der } \\
\text { ausgeschn. } \\
\text { Papierstücke }\end{array}$ \\
\hline $\begin{array}{r}1 \\
2 \\
3 \\
4 \\
5 \\
6 \\
7 \\
8 \\
9 \\
10 \\
11 \\
12 \\
13 \\
14 \\
15 \\
16 \\
17 \\
18 \\
19 \\
20 \\
21 \\
22 \\
23 \\
24 \\
25 \\
26 \\
27 \\
28 \\
29 \\
30 \\
31 \\
32 \\
33 \\
34 \\
35 \\
36 \\
37 \\
38 \\
39\end{array}$ & $\begin{array}{r}4 \\
5 \\
5 \\
4 \\
4 \\
7 \\
7 \\
7 \\
10 \\
10 \\
10 \\
13 \\
16 \\
16 \\
15 \\
15 \\
15 \\
15 \\
19 \\
17 \\
17 \\
15 \\
15 \\
17 \\
15 \\
15 \\
17 \\
13 \\
12 \\
11 \\
14 \\
8 \\
9 \\
6 \\
3 \\
3 \\
2 \\
2 \\
2\end{array}$ & 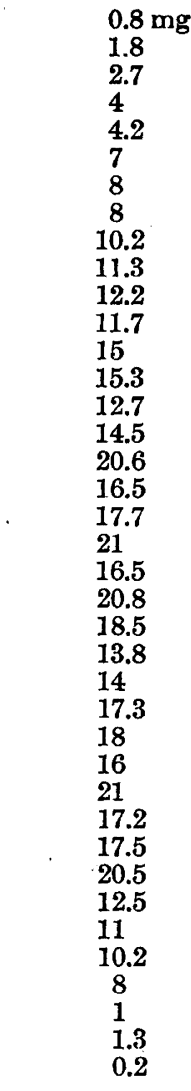 \\
\hline & & 470.5 \\
\hline
\end{tabular}

$\mathrm{V}$ (in $\left.\pi \mathrm{r}^{2} \mathrm{qmm}\right)=0.1870708 \mathrm{cmm}$.

$V($ in $1 \mathrm{gcm})=0.5359 \mathrm{cmm}$.

Ausgleichsdicke $=0.005359 \mathrm{~mm}$

(rund $5.4 \mu$ ). 
Tabelle 29.

Muskel. Rückenhaut.

\begin{tabular}{|c|c|c|}
\hline $\begin{array}{l}\text { Schnitt- } \\
\text { nummer }\end{array}$ & $\begin{array}{l}\text { Zahl der } \\
\text { Muskel- } \\
\text { stücke }\end{array}$ & $\begin{array}{l}\text { Gewicht der } \\
\text { ausgeschn. } \\
\text { Papierstücke }\end{array}$ \\
\hline 1 & 2 & $0.2 \mathrm{mg}$ \\
\hline 2 & 8 & 4 \\
\hline 3 & 11 & 9 \\
\hline 4 & 14 & 9 \\
\hline 5 & 19 & 14.3 \\
\hline 6 & 21 & 17 \\
\hline 7 & 24 & 24 \\
\hline 8 & 25 & 26.5 \\
\hline 9 & 26 & 27.5 \\
\hline 10 & 24 & 34 \\
\hline 11 & 22 & 30.5 \\
\hline 12 & 21 & 27.5 \\
\hline 13 & 18 & 23 \\
\hline 14 & 22 & 28.5 \\
\hline 15 & 21 & 28 \\
\hline 16 & 19 & 30.5 \\
\hline 17 & 19 & 28 \\
\hline 18 & 18 & 30.2 \\
\hline 19 & 19 & 33.5 \\
\hline 20 & 19 & 34 \\
\hline 21 & 18 & 34.8 \\
\hline 22 & 18 & 38.2 \\
\hline 23 & 18 & 38 \\
\hline 24 & 13 & 41.2 \\
\hline 25 & 15 & 52 \\
\hline 26 & 15 & 60.2 \\
\hline 27 & 15 & 49 \\
\hline 28 & 13 & 56.3 \\
\hline 29 & 12 & 39 \\
\hline 30 & 10 & 34 \\
\hline 31 & 10 & 19 \\
\hline 32 & 9 & 17.2 \\
\hline 33 & 7 & 12 \\
\hline 34 & 6 & 8.5 \\
\hline 35 & 8 & 9 \\
\hline 36 & 8 & 8 \\
\hline 37 & 8 & 6.5 \\
\hline 38 & 7 & 4.5 \\
\hline 39 & 6 & 3 \\
\hline 40 & 5 & 2 \\
\hline
\end{tabular}

\begin{tabular}{|c|c|c|}
\hline $\begin{array}{c}\text { Schnitt- } \\
\text { nummer }\end{array}$ & $\begin{array}{c}\text { Zahl der } \\
\text { Muskel- } \\
\text { střcke }\end{array}$ & $\begin{array}{c}\text { Gewicht der } \\
\text { ausgeschn. } \\
\text { Papierstücke }\end{array}$ \\
\hline 41 & 5 & $1.5 \mathrm{mg}$ \\
42 & 5 & 2 \\
43 & 5 & 2 \\
44 & 5 & 2.5 \\
45 & 4 & 2 \\
46 & 4 & 2 \\
47 & 4 & 2.2 \\
48 & 4 & 2 \\
49 & 3 & 2 \\
50 & 3 & 2 \\
51 & 3 & 2.2 \\
52 & 3 & 2.2 \\
53 & 3 & 2 \\
54 & 2 & 1.2 \\
55 & 2 & 0.8 \\
56 & 2 & 0.8 \\
57 & 2 & 0.5 \\
58 & 2 & 0.2 \\
\hline & & 1021.7 \\
& & \\
\hline & & \\
\hline & & \\
\hline
\end{tabular}

$\mathrm{V}$ (in $\left.\pi \mathrm{r}^{2} \mathrm{gmm}\right)=0.40622792 \mathrm{cmm}$. $V($ in $1 \mathrm{gcm})=1.1638 \mathrm{cmm}$.

Ausgleichsdicke $=0.011638 \mathrm{~mm}$

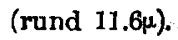


Tabelle 30 .

Muskel. Oberarm

(Streckseite).

\begin{tabular}{|c|c|c|}
\hline $\begin{array}{l}\text { Schnitt- } \\
\text { nummer }\end{array}$ & $\begin{array}{c}\text { Zahl der } \\
\text { Muskel- } \\
\text { stücke }\end{array}$ & $\begin{array}{l}\text { Gewicht der } \\
\text { ausgeschn. } \\
\text { Papierstücke }\end{array}$ \\
\hline 1 & 10 & $11 \mathrm{mg}$ \\
\hline 2 & 17 & 16 \\
\hline 3 & 17 & 31.5 \\
\hline 4 & 22 & 36 \\
\hline 5 & 28 & 45.5 \\
\hline 6 & 25 & 44 \\
\hline 7 & 31 & 67 \\
\hline 8 & 29 & 63.5 \\
\hline 9 & 26 & 58 \\
\hline 10 & 28 & 67 \\
\hline 11 & 28 & 62 \\
\hline 12 & 30 & 92.5 \\
\hline 13 & 29 & 114.5 \\
\hline 14 & 30 & 127 \\
\hline 15 & 27 & 101 \\
\hline 16 & 26 & 81 \\
\hline 17 & 19 & 64.5 \\
\hline 18 & 14 & 66 \\
\hline 19 & 10 & 63.5 \\
\hline 20 & 8 & 47 \\
\hline 21 & 4 & 12 \\
\hline 22 & 2 & 3 \\
\hline & & 1273.5 \\
\hline
\end{tabular}

$\mathrm{V}$ (in $\left.\pi \mathrm{r}^{2} \mathrm{qmm}\right)-0.5063436 \mathrm{cmm}$.

$\mathrm{V}$ (in $1 \mathrm{qcm})=1.4506 \mathrm{cmm}$.

Ausgleichsdicke $=0.014506 \mathrm{~mm}$

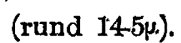

Tabelle 31.

Muskel. Vorderarm

(Streckseite).

\begin{tabular}{|c|c|c|}
\hline $\begin{array}{l}\text { Schnitt- } \\
\text { nummer }\end{array}$ & $\begin{array}{l}\text { Zahl der } \\
\text { Muskel- } \\
\text { stücke }\end{array}$ & $\begin{array}{l}\text { Gewicht der } \\
\text { ausgeschn. } \\
\text { Papierstücke }\end{array}$ \\
\hline 1 & 1 & $0.5 \mathrm{mg}$ \\
\hline 2 & 1 & 0.5 \\
\hline 3 & 2 & 2 \\
\hline 4 & 3 & 3 \\
\hline 5 & 4 & 8 \\
\hline 6 & 6 & 10 \\
\hline 7 & 9 & 11 \\
\hline 8 & 13 & 17.5 \\
\hline 9 & 18 & 14.5 \\
\hline 10 & 20 & 22.3 \\
\hline 11 & 24 & 37.7 \\
\hline 12 & 24 & 31 \\
\hline 13 & 25 & 29.3 \\
\hline 14 & 26 & 41.5 \\
\hline 15 & 24 & 45 \\
\hline 16 & 25 & 61 \\
\hline 17 & 22 & 69 \\
\hline 18 & 19 & 83.5 \\
\hline 19 & 17 & 87.5 \\
\hline 20 & 18 & 67.5 \\
\hline 21 & 14 & 54 \\
\hline 22 & 16 & 60 \\
\hline 23 & 20 & 78 \\
\hline 24 & 11 & 105 \\
\hline 25 & 13 & 113 \\
\hline 26 & 7 & 109.5 \\
\hline 27 & 6 & 30 \\
\hline 28 & 1 & 0.5 \\
\hline 29 & 1 & 7.2 \\
\hline 30 & 1 & 5 \\
\hline 31 & 2 & 5 \\
\hline \multicolumn{3}{|c|}{1209.5} \\
\hline
\end{tabular}

$\mathrm{V}$ (in $\left.\pi \mathrm{r}^{2} \mathrm{qmm}\right)=0.4808972 \mathrm{cmm}$.

$V($ in $1 \mathrm{gcm})=1.3777 \mathrm{cmm}$.

Ausgleichsdicke $=0.013777 \mathrm{~mm}$

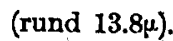


Tabelle 32.

Muskel. Oberschenkel (lateral.)

\begin{tabular}{|c|c|c|}
\hline $\begin{array}{l}\text { Schnitt- } \\
\text { nummer }\end{array}$ & $\begin{array}{l}\text { Zahl der } \\
\text { Muskel- } \\
\text { stücke }\end{array}$ & $\begin{array}{l}\text { Gewicht der } \\
\text { ausgeschn. } \\
\text { Papierstücke }\end{array}$ \\
\hline $\begin{array}{r}1 \\
2 \\
3 \\
4 \\
\mathbf{5} \\
6 \\
7 \\
8 \\
9 \\
10 \\
11 \\
12 \\
13 \\
14 \\
15 \\
16 \\
17 \\
18 \\
19 \\
20 \\
21 \\
22 \\
23 \\
\mathbf{2 4} \\
25 \\
26 \\
27 \\
28 \\
29 \\
30 \\
31 \\
32 \\
33 \\
34 \\
35 \\
36 \\
37 \\
38 \\
39 \\
40 \\
41 \\
42 \\
43 \\
44 \\
45 \\
46 \\
47\end{array}$ & $\begin{array}{r}1 \\
2 \\
3 \\
5 \\
5 \\
8 \\
8 \\
8 \\
9 \\
9 \\
15 \\
13 \\
15 \\
14 \\
17 \\
18 \\
17 \\
20 \\
20 \\
22 \\
22 \\
24 \\
26 \\
23 \\
22 \\
18 \\
19 \\
18 \\
20 \\
17 \\
17 \\
13 \\
11 \\
11 \\
8 \\
8 \\
8 \\
8 \\
9 \\
7 \\
6 \\
8 \\
6 \\
4 \\
3 \\
3 \\
1\end{array}$ & $\begin{array}{l}0.3 \mathrm{mg} \\
1 \\
1 \\
2.5 \\
6 \\
7 \\
9.2 \\
8 \\
8.3 \\
9 \\
15.7 \\
15 \\
23 \\
23 \\
32 \\
40.8 \\
38.2 \\
43 \\
45 \\
56 \\
60 \\
63 \\
71 \\
85.5 \\
84 \\
84.3 \\
76 \\
84 \\
85 \\
92 \\
74 \\
51 \\
51.5 \\
53.5 \\
45 \\
39 \\
48 \\
51.5 \\
37 \\
32.8 \\
27.8 \\
20.2 \\
12.3 \\
8.5 \\
2.8 \\
5.2 \\
0.3 \\
\end{array}$ \\
\hline & & 1729.2 \\
\hline
\end{tabular}

$\mathrm{V}$ (in $\left.\pi \mathrm{r}^{2} \mathrm{gmm}\right)=0.68752992 \mathrm{cmm}$.

$\mathrm{V}$ (in $1 \mathrm{gcm}$ ) $=1.9700 \mathrm{cmm}$.

Ausgleichsdicke $=0.0197 \mathrm{~mm}$ (rund 19.7 $\mu$ ).
Tabelle 33.

Muskel. Unterschenkel (lateral).

\begin{tabular}{|c|c|c|}
\hline $\begin{array}{l}\text { Schnitt- } \\
\text { nummer }\end{array}$ & $\begin{array}{l}\text { Zahl der } \\
\text { Muskel- } \\
\text { stäcke }\end{array}$ & $\begin{array}{l}\text { Gewicht der } \\
\text { ausgeschn. } \\
\text { Papierstücke }\end{array}$ \\
\hline 1 & 1 & $0.8 \mathrm{mg}$ \\
\hline 2 & 1 & 0.5 \\
\hline 3 & 1 & 0.3 \\
\hline 4 & 4 & 2.2 \\
\hline 5 & 9 & 9.2 \\
\hline 6 & 18 & 10.5 \\
\hline 7 & 27 & 15 \\
\hline 8 & 30 & 25 \\
\hline 9 & 29 & 34.5 \\
\hline 10 & 35 & 53 \\
\hline 11 & 26 & 56 \\
\hline 12 & 28 & 60.2 \\
\hline 13 & 27 & 72 \\
\hline 14 & 27 & 78.2 \\
\hline 15 & 23 & 85.5 \\
\hline 16 & 28 & 103 \\
\hline 17 & 28 & 119.5 \\
\hline 18 & 29 & 126 \\
\hline 19 & 25 & 159.7 \\
\hline 20 & 23 & 169 \\
\hline 21 & 19 & 190.5 \\
\hline 22 & 18 & 168.5 \\
\hline 23 & 15 & 114.5 \\
\hline 24 & 11 & 73 \\
\hline 25 & 7 & 55.5 \\
\hline 26 & 6 & 31.8 \\
\hline 27 & 5 & 22 \\
\hline 28 & 3 & 4.5 \\
\hline & & 1840.4 \\
\hline
\end{tabular}

$V\left(\right.$ in $\left.\pi \mathrm{r}^{2} \mathrm{gmm}\right)=0.73174304 \mathrm{cmm}$.

$\mathrm{V}$ (in $1 \mathrm{gcm}$ ) $=2.0963 \mathrm{cmm}$.

Ausgleichsdicke $=0.020963 \mathrm{~mm}$ (rund 21 $\mu$ ). 
Tabelle 34.

Volumen der Muskelindividuen in Stirnhaut.

\begin{tabular}{|c|c|c|c|c|c|}
\hline $\begin{array}{c}\text { Muskel- } \\
\text { nummer }\end{array}$ & $\begin{array}{c}\text { Haarzahl } \\
\text { in einer } \\
\text { 'Gruppe }\end{array}$ & Schnittzahl & $\begin{array}{c}\text { Zahl der } \\
\text { Muskel- } \\
\text { stücke }\end{array}$ & $\begin{array}{c}\text { Gewicht der } \\
\text { ausgeschn. } \\
\text { Papierstücke }\end{array}$ & $\begin{array}{c}\text { Volumen des } \\
\text { Muskelindiv. }\end{array}$ \\
\hline a & 6 & 21 & 56 & $43.5 \mathrm{mg}$ & $0.0216195 \mathrm{cmm}$ \\
b & 6 & 22 & 49 & 58 & 0.0288260 \\
c & 6 & 22 & 55 & 31 & 0.0154070 \\
d & 6 & 23 & 52 & 54.3 & 0.0269871 \\
e & 4 & 17 & 21 & 21.5 & 0.0106855 \\
f & 4 & 19 & 35 & 21 & 0.0104370 \\
g & 4 & 21 & 37 & 27 & 0.0134190 \\
h & 3 & 16 & 30 & 20 & 0.0099400 \\
i & 3 & 20 & 37 & 22.5 & 0.0111825 \\
j & 3 & 18 & 21 & 14 & 0.0069580 \\
\hline \multicolumn{2}{|r|}{ durchschnittlich } & & & 0.0155462 \\
\hline
\end{tabular}

Tabelle 35.

Volumen der Muskelindividuen in Hinterhaupthaut.

\begin{tabular}{|c|c|c|c|c|c|}
\hline $\begin{array}{c}\text { Muskel- } \\
\text { nummer }\end{array}$ & $\begin{array}{c}\text { Haarzahl } \\
\text { in einer } \\
\text { Gruppe }\end{array}$ & Schnittzahl & $\begin{array}{c}\text { Zahl der } \\
\text { Muskel- } \\
\text { stücke }\end{array}$ & $\begin{array}{c}\text { Gewicht der } \\
\text { ausgeschn. } \\
\text { Papierstücke }\end{array}$ & $\begin{array}{c}\text { Volumen des } \\
\text { Muskelindiv. }\end{array}$ \\
\hline a & $\mathbf{6}$ & 31 & 77 & $91 \mathrm{mg}$ & $0.0452270 \mathrm{cmm}$ \\
b & 6 & 23 & 60 & 53 & 0.0263410 \\
c & 5 & 26 & 47 & 54 & 0.0268380 \\
d & 4 & 34 & 74 & 94 & 0.0467180 \\
e & 4 & 31 & 92 & 97.5 & 0.0484575 \\
f & $\mathbf{3}$ & 27 & 48 & 32 & 0.0159040 \\
g & 3 & 30 & 55 & 32 & 0.0159040 \\
h & $\mathbf{3}$ & 25 & 35 & 22.5 & 0.0111825 \\
i & 3 & 27 & 61 & 36.5 & 0.0181405 \\
j & $\mathbf{3}$ & 18 & 22 & 24 & 0.0119280 \\
\hline \multicolumn{2}{|r|}{ durchschnittlich } & & & 0.0216641 \\
\hline
\end{tabular}

Tabelle 36.

Volumen der Muskelindividuen in Bauchhaut.

\begin{tabular}{|c|c|c|c|c|c|}
\hline $\begin{array}{c}\text { Muskel- } \\
\text { nummer }\end{array}$ & $\begin{array}{c}\text { Haarzahl } \\
\text { in einer } \\
\text { Gruppe }\end{array}$ & Schnittzahl & $\begin{array}{c}\text { Zahl der } \\
\text { Muskel- } \\
\text { stücke }\end{array}$ & $\begin{array}{c}\text { Gewicht der } \\
\text { ausgeschn. } \\
\text { Papierstücke }\end{array}$ & $\begin{array}{c}\text { Volumen des } \\
\text { Muskelindiv. }\end{array}$ \\
\hline a & 3 & 21 & 66 & $106 \mathrm{mg}$ & $0.0421456 \mathrm{cmm}$ \\
b & 2 & 20 & 40 & 25 & 0.0099400 \\
c & 2 & 21 & 36 & 32 & 0.0131208 \\
d & 2 & 23 & 41 & 43.5 & 0.0172956 \\
e & 2 & 22 & 34 & 51 & 0.0202776 \\
f & 2 & 17 & 26 & 25 & 0.0099400 \\
g & 2 & 21 & 35 & 64 & 0.0254464 \\
h & 2 & 17 & 26 & 17 & 0.0067592 \\
i & 2 & 23 & 75 & 81 & 0.0322056 \\
j & 2 & 20 & 24 & 29.5 & 0.0117292 \\
\hline \multicolumn{2}{|r|}{ durchschnittlich } & & & 0.0188860 \\
\hline
\end{tabular}


Tabelle 37.

Volumen der Muskelindividuen in Rückenhaut.

\begin{tabular}{|c|c|c|c|c|c|}
\hline $\begin{array}{c}\text { Muskel- } \\
\text { nummer }\end{array}$ & $\begin{array}{c}\text { Haarzahl } \\
\text { in einer } \\
\text { Gruppe }\end{array}$ & Schnittzahl & $\begin{array}{c}\text { Zahl der } \\
\text { Muskel- } \\
\text { stücke }\end{array}$ & $\begin{array}{c}\text { Gewicht der } \\
\text { ausgeschn. } \\
\text { Papierstücke }\end{array}$ & $\begin{array}{c}\text { Volumen des } \\
\text { Muskelindiv. }\end{array}$ \\
\hline a & 4 & 35 & 123 & $138 \mathrm{mg}$ & $0.0548688 \mathrm{cmm}$ \\
$\mathrm{b}$ & 3 & 36 & 53 & 110 & 0.0437360 \\
$\mathrm{c}$ & 3 & 36 & 108 & 156.5 & 0.0622244 \\
d & 3 & 46 & 84 & 184 & 0.0731584 \\
e & 2 & 27 & 68 & 111.5 & 0.0443324 \\
f & 2 & 35 & 57 & 142 & 0.0564592 \\
g & 2 & 29 & 58 & 47 & 0.0186872 \\
h & 2 & 58 & 139 & 183.8 & 0.0730789 \\
i & 2 & 22 & 44 & 42 & 0.0166992 \\
j & 2 & 23 & 45 & 87.5 & 0.0347900 \\
\hline \multicolumn{2}{|l}{ durchschnittlich } & & & 0.0478034 \\
\hline
\end{tabular}

Tabelle 38.

Volumen der Muskelindividuen in Oberarm (Streckseite).

\begin{tabular}{|c|c|c|c|c|c|}
\hline $\begin{array}{c}\text { Muskel- } \\
\text { nummer }\end{array}$ & $\begin{array}{c}\text { Haarzahl } \\
\text { in einer } \\
\text { Gruppe }\end{array}$ & Schnittzahl & $\begin{array}{c}\text { Zahl der } \\
\text { Muskel- } \\
\text { stïcke }\end{array}$ & $\begin{array}{c}\text { Gewicht der } \\
\text { ausgeschn. } \\
\text { Papierstücke }\end{array}$ & $\begin{array}{c}\text { Volumen des } \\
\text { Muskelindiv. }\end{array}$ \\
\hline a & 3 & 12 & 47 & $186 \mathrm{mg}$ & $0.0739536 \mathrm{cmm}$ \\
b & 3 & 14 & 72 & 196.5 & 0.0781284 \\
c & 3 & 16 & 53 & 107 & 0.0425432 \\
d & 3 & 17 & 40 & 140 & 0.0556640 \\
e & 3 & 22 & 82 & 192 & 0.0763392 \\
f & 2 & 16 & 49 & 86.5 & 0.0343924 \\
g & 2 & 13 & 36 & 117 & 0.0465192 \\
h & 2 & 14 & 44 & 105.5 & 0.0419468 \\
i & 2 & 14 & 66 & 167.5 & 0.0665980 \\
j & 2 & 14 & 38 & 137 & 0.0544712 \\
\hline \multicolumn{2}{|r|}{ durchschnittlich } & & & 0.0570556 \\
\hline
\end{tabular}

Tabelle 39.

Volumen der Muskelindividuen in Vorderarm (Streckseite).

\begin{tabular}{|c|c|c|c|c|c|}
\hline $\begin{array}{c}\text { Muskel- } \\
\text { nummer }\end{array}$ & $\begin{array}{c}\text { Haarzahl } \\
\text { in einer } \\
\text { Gruppe }\end{array}$ & Schnittzahl & $\begin{array}{c}\text { Zahl der' } \\
\text { Muskel- } \\
\text { stücke }\end{array}$ & $\begin{array}{c}\text { Gewicht der } \\
\text { ausgeschn. } \\
\text { Papierstücke }\end{array}$ & $\begin{array}{c}\text { Volumen des } \\
\text { Muskelindiv. }\end{array}$ \\
\hline a & 4 & 20 & 50 & $142.8 \mathrm{mg}$ & $0.0567773 \mathrm{cmm}$ \\
b & 3 & 20 & 67 & 223 & 0.0886648 \\
c & 3 & 40 & 96 & 187.5 & 0.0745500 \\
d & 3 & 12 & 63 & 296.5 & 0.1178884 \\
e & 3 & 12 & 36 & 90.5 & 0.0359828 \\
f & 2 & 19 & 42 & 128.8 & 0.0512109 \\
g & 2 & 21 & 42 & 194 & 0.0369768 \\
h & 2 & 14 & 51 & 17.5 & 0.0771344 \\
i & 2 & 14 & 23 & 147 & 0.0584472 \\
j & 2 & 15 & 36 & & 0.0604591 \\
\hline
\end{tabular}


Tabelle 40 .

Volumen der Muskelindividuen in Oberschenkel (lateral).

\begin{tabular}{|c|c|c|c|c|c|}
\hline $\begin{array}{c}\text { Muskel- } \\
\text { nummer }\end{array}$ & $\begin{array}{c}\text { Haarzahl } \\
\text { in einer } \\
\text { Gruppe }\end{array}$ & Schnittzahl & $\begin{array}{c}\text { Zahl der } \\
\text { Muskel- } \\
\text { stücke }\end{array}$ & $\begin{array}{c}\text { Gewicht der } \\
\text { ausgeschn. } \\
\text { Papierstücke }\end{array}$ & $\begin{array}{c}\text { Volumen des } \\
\text { Muskelindiv. }\end{array}$ \\
\hline a & 3 & 36 & 86 & $432.5 \mathrm{mg}$ & $0.1719620 \mathrm{cmm}$ \\
b & 3 & 21 & 42 & 187.2 & 0.0744307 \\
c & 2 & 33 & 80 & 196 & 0.0779296 \\
d & 2 & 32 & 59 & 118 & 0.0469168 \\
e & 2 & 33 & 89 & 280.2 & 0.1114075 \\
f & 2 & 19 & 34 & 88 & 0.0349888 \\
g & 2 & 32 & 73 & 331.5 & 0.1338044 \\
h & 2 & 23 & 87 & 240.5 & 0.0956228 \\
i & 2 & 18 & 58 & 209 & 0.0830984 \\
j & 1 & 32 & 65 & 103 & 0.0409528 \\
\hline \multicolumn{2}{|l}{ durchschnittlich } \\
\hline
\end{tabular}

Tabelle 41.

Volumen der Muskelindividuen in Unterschenkel (lateral).

\begin{tabular}{|c|c|c|c|c|c|}
\hline $\begin{array}{c}\text { Muskel- } \\
\text { nummer }\end{array}$ & $\begin{array}{c}\text { Haarzahl } \\
\text { in einer } \\
\text { Gruppe }\end{array}$ & Schnittzahl & $\begin{array}{c}\text { Zahl der } \\
\text { Muskel- } \\
\text { stäcke }\end{array}$ & $\begin{array}{c}\text { Gewicht der } \\
\text { ausgeschn. } \\
\text { Papierstücke }\end{array}$ & Volumen des \\
\hline a & 3 & 22 & 61 & $295 \mathrm{mg}$ & $0.1172920 \mathrm{cmm}$ \\
b & 3 & 31 & 119 & 368 & 0.1463168 \\
c & 3 & 18 & 69 & 586 & 0.2329936 \\
d & 2 & 20 & 30 & 93.5 & 0.0371756 \\
e & 2 & 23 & 80 & 192.5 & 0.0765380 \\
f & 2 & 8 & 21 & 115.5 & 0.0459228 \\
g & 2 & 19 & 58 & 229.5 & 0.0912492 \\
h & 2 & 14 & 57 & 211.8 & 0.0842117 \\
i & 2 & 25 & 80 & 175 & 0.0695800 \\
j & 2 & 12 & 21 & 139 & 0.0552664 \\
\hline durchschnittlich & & & \\
\hline
\end{tabular}




\section{Tabellarische Zusammenstellung.}

Im folgenden möchte ich die obigen Befunde noch einmal tabellarisch zusammenstellen.

1. Schweissdrüse. teilen.

a. Menge und Ausmündungszahl der Drüse in den einzelnen Körper-

Tabelle I. (Schweissdrüse).

\begin{tabular}{|l|c|c|c|c|}
\hline Körperteile & $\begin{array}{c}\text { Volumen der } \\
\begin{array}{c}\text { Schweissdrüse in } \\
\text { 1 qcm Haut- } \\
\text { flächeninhalt }\end{array}\end{array}$ & $\begin{array}{c}\text { Angenommene } \\
\text { Ausgleichsdicke } \\
\text { (rund) }\end{array}$ & $\begin{array}{c}\text { Angenommene } \\
\text { Volumen des } \\
\text { Schweissdrüsen- } \\
\text { individuums }\end{array}$ & $\begin{array}{c}\text { Ausmündungs- } \\
\text { zahl der } \\
\text { Schweissdrüse } \\
\text { in 1 qcm }\end{array}$ \\
\hline Stirn & $12.6437 \mathrm{cmm}$ & $126.4 \mu$ & $0.038548 \mathrm{cmm}$ & 328 \\
Hinterhaupt & 6.7741 & 67.7 & 0.022431 & 302 \\
Bauch & 4.1313 & 41.3 & 0.025345 & 163 \\
Rücken & 4.5854 & 45.9 & 0.029970 & 153 \\
Oberarm (Ss) & 2.9923 & 29.9 & 0.021073 & 142 \\
Vorderarm (Ss) & 4.0118 & 40.1 & 0.032616 & 123 \\
Obersch (lat.) & 3.9952 & 40. & 0.031213 & 128 \\
Untersch. (lat.) & 5.3292 & 53.3 & 0.033729 & 158 \\
\hline
\end{tabular}

$\mathrm{Ss}=$ Streckseite.

b. Reihenfolge der Mengengrösse und Ausmündungszahl der Drüse in einzelnen Körperteilen.

Tabelle II. (Schweissdrüse, Reihenfolge).

\begin{tabular}{|c|c|c|c|c|c|}
\hline \multicolumn{2}{|c|}{$\begin{array}{c}\text { Volumen der Schweissdrüse } \\
\text { in } 1 \mathrm{gcm}\end{array}$} & \multicolumn{2}{|c|}{$\begin{array}{l}\text { Volumen des angenommenen } \\
\text { Drüsenindividuums }\end{array}$} & \multicolumn{2}{|c|}{$\begin{array}{l}\text { Ausmündüngszahl der } \\
\text { Schweissdrüse in } 1 \mathrm{gcm}\end{array}$} \\
\hline Körperteile & Volumen & Körperteile & Volumen & Körperteile & Zahl \\
\hline Stirn & $12.6437 \mathrm{cmm}$ & Stirn & $0.038548 \mathrm{cmm}$ & Stirn & 328 \\
\hline Hinterhaupt & 6.7741 & Untersch. (lat.) & 0.033729 & Hinterhaupt & 302 \\
\hline Untersch. (lat.) & 5.3292 & Vorderarm (Ss) & 0.032616 & Bauch & 163 \\
\hline Rücken & 4.5854 & Obersch. (lat.) & 0.031213 & Untersch. (lat.) & 158 \\
\hline Bauch & 4.1313 & Rücken & 0.029970 & Rücken & 153 \\
\hline Vorderarm (Ss) & 4.0118 & Bauch & 0.025345 & Oberarm (Ss) & 142 \\
\hline Obersch. (lat.) & 3.9952 & Hinterhaupt & 0.022431 & Obersch. (lat.) & 128 \\
\hline Oberarm (Ss) & 2.9923 & Oberarm (Ss) & 0.021073 & Vorderarm' (Ss) & 123 \\
\hline
\end{tabular}


c. Menge und Ausmündungszahl der Drüse in den Hauptkörperabschnitten.

Tabelle III. (Schweissdrüse).

\begin{tabular}{|c|c|c|c|c|c|c|}
\hline \multirow[t]{2}{*}{ Körperteile } & \multicolumn{2}{|c|}{$\begin{array}{l}\text { Volumen der Schweiss- } \\
\text { drüse in } 1 \mathrm{qcm}\end{array}$} & \multicolumn{2}{|c|}{$\begin{array}{l}\text { Volumen der angenomme- } \\
\text { nen Drüsenindividuen }\end{array}$} & \multicolumn{2}{|c|}{$\begin{array}{l}\text { Ausmündungszahl } \\
\text { der Schweissdrüse } \\
\text { in } 1 \mathrm{gcm}\end{array}$} \\
\hline & Körperteile & Volumen & Körperteile & Volumen & Körperteile & Zahl \\
\hline ganzer Körper & $\begin{array}{l}\text { Kopf } \\
\text { Rumpf } \\
\text { Extremität }\end{array}$ & $\begin{array}{l}9.7089 \mathrm{cmm} \\
4.3584 \\
4.0821\end{array}$ & $\begin{array}{l}\text { Kopf } \\
\text { Extremität } \\
\text { Rumpf }\end{array}$ & $\begin{array}{l}0.030490 \mathrm{cmm} \\
0.029658 \\
0.027658\end{array}$ & $\begin{array}{l}\text { Kopf } \\
\text { Rumpf } \\
\text { Extremität }\end{array}$ & $\begin{array}{l}315 \\
153 \\
138\end{array}$ \\
\hline Kopf & $\begin{array}{l}\text { Stirn } \\
\text { Hinterhaupt }\end{array}$ & $\begin{array}{l}12.6437 \\
6.7741\end{array}$ & $\begin{array}{l}\text { Stirn } \\
\text { Hinterhaupt }\end{array}$ & $\begin{array}{l}0.038548 \\
0.022431\end{array}$ & $\begin{array}{l}\text { Stirn } \\
\text { Hinterhaupt }\end{array}$ & $\begin{array}{l}328 \\
302\end{array}$ \\
\hline Rumpf & $\begin{array}{l}\text { Rücken } \\
\text { Bauch }\end{array}$ & $\begin{array}{l}4.5854 \\
4.1313\end{array}$ & $\begin{array}{l}\text { Rücken } \\
\text { Bauch }\end{array}$ & $\begin{array}{l}0.029970 \\
0.025345\end{array}$ & $\begin{array}{l}\text { Bauch } \\
\text { Rücken }\end{array}$ & $\begin{array}{l}163 \\
153\end{array}$ \\
\hline $\begin{array}{l}\text { Extremität } \\
\text { (a) }\end{array}$ & $\begin{array}{l}\text { untere Extr. } \\
\text { obere Extr. }\end{array}$ & $\begin{array}{l}4.6622 \\
3.5021\end{array}$ & $\begin{array}{l}\text { untere Extr. } \\
\text { obere Extr. }\end{array}$ & $\begin{array}{l}0.032471 \\
0.026845 \\
\end{array}$ & $\begin{array}{l}\text { untere Extr. } \\
\text { obere Extr. }\end{array}$ & $\begin{array}{l}143 \\
133\end{array}$ \\
\hline $\begin{array}{l}\text { Extremität } \\
\text { (b) }\end{array}$ & $\begin{array}{l}\text { Distalis } \\
\text { Proximalis }\end{array}$ & $\begin{array}{l}4.6705 \\
\mathbf{3 . 4 9 3 7}\end{array}$ & $\begin{array}{l}\text { Distalis } \\
\text { Proximalis }\end{array}$ & $\begin{array}{l}0.033173 \\
0.026143\end{array}$ & $\begin{array}{l}\text { Distalis } \\
\text { Proximalis }\end{array}$ & $\begin{array}{l}141 \\
135\end{array}$ \\
\hline obere Extr. & $\begin{array}{l}\text { Vorderarm } \\
\text { Oberarm }\end{array}$ & $\begin{array}{l}4.0118 \\
2.9923\end{array}$ & $\begin{array}{l}\text { Vorderarm } \\
\text { Oberarm }\end{array}$ & $\begin{array}{l}0.032616 \\
0.021073\end{array}$ & $\begin{array}{l}\text { Oberarm } \\
\text { Vorderarm }\end{array}$ & $\begin{array}{l}142 \\
123\end{array}$ \\
\hline untere Extr. & $\begin{array}{l}\text { Untersch. } \\
\text { Oberseh. }\end{array}$ & $\begin{array}{l}5.3292 \\
3.9952\end{array}$ & $\begin{array}{l}\text { Untersch. } \\
\text { Obersch. }\end{array}$ & $\begin{array}{l}0.033729 \\
0.031213\end{array}$ & $\begin{array}{l}\text { Untersch. } \\
\text { Obersch. }\end{array}$ & $\begin{array}{l}158 \\
128\end{array}$ \\
\hline
\end{tabular}




\section{Talgdrüse.}

a. Menge der Drüse in den einzelnen Körperteilen.

Tabelle IV. (Talgdrüse).

\begin{tabular}{|c|c|c|c|c|c|}
\hline \multirow[b]{2}{*}{ Körperteile } & \multirow{2}{*}{$\begin{array}{l}\text { Volumen } \\
\text { der Talg- } \\
\text { drüse in } \\
1 \mathrm{gcm}\end{array}$} & \multirow{2}{*}{$\begin{array}{c}\text { Angenom- } \\
\text { mene Aus- } \\
\text { gleichsdicke } \\
\text { (rund) }\end{array}$} & \multirow{2}{*}{$\begin{array}{c}\text { Volumen der } \\
\text { Talgdrüsen- } \\
\text { individuen } \\
\text { (durchschn.) }\end{array}$} & \multicolumn{2}{|c|}{ Reihenfolge nach Volumen } \\
\hline & & & & in $1 \mathrm{qcm}$ & $\begin{array}{l}\text { Drüsenindivi- } \\
\text { duum (durch- } \\
\text { schnittlich) }\end{array}$ \\
\hline Stirn & $9.2911 \mathrm{cmm}$ & $92.9 \mu$ & $0.065256 \mathrm{cmm}$ & Stirn & Hinterhaupt \\
\hline Hinterhaupt & 9.2498 & 92.5 & 0.070137 & Hinterhaupt & Stirn \\
\hline Bauch & 0.1601 & 1.6 & 0.005857 & Rücken & Rücken \\
\hline Rücken & 0.4643 & 4.6 & 0.018866 & Vorderarm (Ss) & Vorderarm $(\mathrm{Ss})$ \\
\hline Oberarm (Ss) & 0.1686 & 1.7 & 0.007368 & Oberarm (Ss) & Oberarm (Ss) \\
\hline Vorderarm (Ss) & 0.3599 & 3.6 & 0.016628 & Bauch & Bauch \\
\hline Obersch. (lat.) & 0.0883 & 0.9 & 0.004493 & Obersch. (lat.) & Obersch. (lat.) \\
\hline Untersch. (lat.) & 0.0424 & 0.4 & 0.002358 & Unterseh. (lat.) & Untersch. (lat.) \\
\hline
\end{tabular}

b. Menge der Drüse in den Hauptkörperabschnitten.

Tabelle V. (Talgdrüse).

\begin{tabular}{|c|c|c|c|c|}
\hline \multirow{2}{*}{ Körperteile } & \multicolumn{2}{|c|}{ Volumen der Talgdrüse in $1 \mathrm{gcm}$} & \multicolumn{2}{|c|}{$\begin{array}{c}\text { Volumen der Talgdrüsenindiv. } \\
\text { (durchschnittlich) }\end{array}$} \\
\hline & Körperteile & Volumen & Körperteile & Volumen \\
\hline ganzer Körper & $\begin{array}{l}\text { Kopf } \\
\text { Rumpf } \\
\text { Extremität }\end{array}$ & $\begin{array}{l}9.2705 \mathrm{cmm} \\
0.3122 \\
0.1648\end{array}$ & $\begin{array}{l}\text { Kopf } \\
\text { Rumpf } \\
\text { Extremität }\end{array}$ & $\begin{array}{l}0.067697 \mathrm{cmm} \\
0.012362 \\
0.007712\end{array}$ \\
\hline Kopf & $\begin{array}{l}\text { Stirn } \\
\text { Hinterhaupt }\end{array}$ & $\begin{array}{l}9.2911 \\
9.2498\end{array}$ & $\begin{array}{l}\text { Hinterhaupt } \\
\text { Stirn }\end{array}$ & $\begin{array}{l}0.070137 \\
0.065256\end{array}$ \\
\hline Rumpf & $\begin{array}{l}\text { Rücken } \\
\text { Bauch }\end{array}$ & $\begin{array}{l}0.4643 \\
0.1601\end{array}$ & $\begin{array}{l}\text { Rücken } \\
\text { Bauch }\end{array}$ & $\begin{array}{l}0.018866 \\
0.005857\end{array}$ \\
\hline $\begin{array}{l}\text { Extremität } \\
\text { (a) }\end{array}$ & $\begin{array}{l}\text { obere Extr. } \\
\text { untere Extr. }\end{array}$ & $\begin{array}{l}0.2643 \\
0.0654\end{array}$ & $\begin{array}{l}\text { obere Extr. } \\
\text { untere Extr. }\end{array}$ & $\begin{array}{l}0.011998 \\
0.006851\end{array}$ \\
\hline $\begin{array}{l}\text { Extremität } \\
\text { (b) }\end{array}$ & $\begin{array}{l}\text { Distalis } \\
\text { Proximalis }\end{array}$ & $\begin{array}{l}0.2012 \\
0.1285\end{array}$ & $\begin{array}{l}\text { Distalis } \\
\text { Proximalis }\end{array}$ & $\begin{array}{l}0.009493 \\
0.005931\end{array}$ \\
\hline obere Extr. & $\begin{array}{l}\text { Vorderarm } \\
\text { Oberarm (Ss) }\end{array}$ & $\begin{array}{l}0.3599 \\
0.1686\end{array}$ & $\begin{array}{l}\text { Vorderarm (Ss) } \\
\text { Oberarm (Ss) }\end{array}$ & $\begin{array}{l}0.016628 \\
0.007368\end{array}$ \\
\hline untere Extr. & $\begin{array}{l}\text { Oberschenkel } \\
\text { Unterschenkel }\end{array}$ & $\begin{array}{l}0.0883 \\
0.0424\end{array}$ & $\begin{array}{l}\text { Oberschenkel } \\
\text { Unterschenkel }\end{array}$ & $\begin{array}{l}0.004493 \\
0.002358\end{array}$ \\
\hline
\end{tabular}




\section{Haarbalgmuskel.}

a. Menge des Muskels in den einzelnen Körperteilen.

Tabelle VI (Muskel).

\begin{tabular}{|c|c|c|c|c|c|}
\hline \multirow[b]{2}{*}{ Körperteile } & \multirow{2}{*}{$\begin{array}{l}\text { Volumen } \\
\text { des } \\
\text { Arrectors } \\
\text { in } 1 \mathrm{gcm}\end{array}$} & \multirow{2}{*}{$\begin{array}{c}\text { Angenom- } \\
\text { mene Aus- } \\
\text { gleichsdicke } \\
\text { (rund) }\end{array}$} & \multirow{2}{*}{$\begin{array}{l}\text { Volumen der } \\
\text { Muskelindiv. } \\
\text { (durchschn.) }\end{array}$} & \multicolumn{2}{|c|}{ Reihenfolge nach Volumen } \\
\hline & & & & in $1 \mathrm{qcm}$ & $\begin{array}{l}\text { Muskelindivi- } \\
\text { duum (durch } \\
\text { schnittlich) }\end{array}$ \\
\hline Stirn & $2.0275 \mathrm{cmm}$ & - $20.3 \mu$ & $0.015546 \mathrm{cmm}$ & Hinterhaupt & Untersch. (lat.) \\
\hline Hinterhaupt & 3.1701 & 31.7 & 0.026664 & Untersch. (lat.) & Obersch. (lat.) \\
\hline Bauch & 0.5359 & 5.4 & 0.018886 & Stirn & Vorderarm (Ss) \\
\hline Rücken & 1.1638 & 11.6 & 0.047803 & Obersch. (lat.) & Oberarm (Ss) \\
\hline Oberarm (Ss) & 1.4506 & 14.5 & 0.057056 & Oberarm (Ss) & Rücken \\
\hline Vorderarm (Ss) & 1.3777 & 13.8 & 0.060459 & Vorderarm (Ss) & Hinterhaupt \\
\hline Obersch. (lat.) & 1.9696 & 19.7 & 0.086911 & Rücken & Bauch \\
\hline Untersch. (lat.) & 2.0963 & 21 & 0.095655 & Bauch & Stirn \\
\hline
\end{tabular}

b. Menge des Muskels in den Hauptkörperabschnitten.

Tabelle VII. (Muskel).

\begin{tabular}{|c|c|c|c|c|}
\hline \multirow{2}{*}{ Körperteile } & \multicolumn{2}{|c|}{ Volumen des Arrectors in $1 \mathrm{gcm}$} & \multicolumn{2}{|c|}{$\begin{array}{l}\text { Volumen der Muskelindiv. } \\
\text { (durchschnittlich) }\end{array}$} \\
\hline & Körperteile & Volumen & Körperteile & Volumen \\
\hline ganzer Körper & $\begin{array}{l}\text { Kopf } \\
\text { Extremität } \\
\text { Rumpf }\end{array}$ & $\begin{array}{l}2.5988 \mathrm{cmm} \\
1.7236 \\
0.8499\end{array}$ & $\begin{array}{l}\text { Extremität } \\
\text { Rumpf } \\
\text { Kopf }\end{array}$ & $\begin{array}{l}0.075020 \mathrm{cmm} \\
0.033345 \\
0.021105\end{array}$ \\
\hline Kopf & $\begin{array}{l}\text { Hinterhaupt } \\
\text { Stirn }\end{array}$ & $\begin{array}{l}3.1701 \\
2.0275\end{array}$ & $\begin{array}{l}\text { Hinterhaupt } \\
\text { Stirn }\end{array}$ & $\begin{array}{l}0.026664 \\
0.015546\end{array}$ \\
\hline Rumpf & $\begin{array}{l}\text { Rücken } \\
\text { Bauch }\end{array}$ & $\begin{array}{l}1.1638 \\
0.5359\end{array}$ & $\begin{array}{l}\text { Rücken } \\
\text { Bauch }\end{array}$ & $\begin{array}{l}0.047803 \\
0.018886\end{array}$ \\
\hline $\begin{array}{l}\text { Extremität } \\
\text { (a) }\end{array}$ & $\begin{array}{l}\text { untere Extr. } \\
\text { obere Extr. }\end{array}$ & $\begin{array}{l}2.0330 \\
1.4142\end{array}$ & $\begin{array}{l}\text { untere Extr. } \\
\text { obere Extr. }\end{array}$ & $\begin{array}{l}0091283 \\
0.058758\end{array}$ \\
\hline $\begin{array}{l}\text { Extremität } \\
\text { (b) }\end{array}$ & $\begin{array}{l}\text { Distalis } \\
\text { Proximalis }\end{array}$ & $\begin{array}{l}1.7370 \\
1.7101\end{array}$ & $\begin{array}{l}\text { Distalis } \\
\text { Proximalis }\end{array}$ & $\begin{array}{l}0.078057 \\
0.071984\end{array}$ \\
\hline obere Extr. & $\begin{array}{l}\text { Oberarm } \\
\text { Vorderarm }\end{array}$ & $\begin{array}{l}1.4506 \\
1.3777\end{array}$ & $\begin{array}{l}\text { Vorderarm } \\
\text { Oberarm }\end{array}$ & $\begin{array}{l}0.060459 \\
0.057056\end{array}$ \\
\hline untere Extr. & $\begin{array}{l}\text { Unterschenkel } \\
\text { Oberschenkel }\end{array}$ & $\begin{array}{l}2.0963 \\
1.9696\end{array}$ & $\begin{array}{l}\text { Unterschenkel } \\
\text { Oberschenkel }\end{array}$ & $\begin{array}{l}0.095655 \\
0.086911\end{array}$ \\
\hline
\end{tabular}




\section{Zusammenfassung.}

Die Mengen der Anhangsorgane der Haut bei dem erwachsenen Deutschen sind in den obigen Schlusstabellen (Tab. I-VII) angegeben. Nun werde ich die wichtigeren Befunde kurz zusammenfassen.

1. Das Volumen der Schweissdrūsen in $\mathbf{1}$ qcm Hautstūck variiert nach den Körperteilen in hohem Masse, wie in Tabelle I und II gezeigt. In den 8 untersuchten Körperteilen ist die Drüsenmenge an der Stirn am grōssten (12.6437 cmm), dann folgen das Hinterhaupt (6.7741 $\mathrm{cmm})$, die Lateralseite des Unterschenkels $(5.3292 \mathrm{cmm})$, der Rücken $(4.5854 \mathrm{cmm})$ u.s.w. ; sie ist am geringsten an der Streckseite des Oberarms $(2.9923 \mathrm{cmm})$. Die relative Schwankungsbreite der Menge beträgt also ca. $4: 1$.

Die Ausmūudungszahl der Schweissdrūsen schwankt auch nach den Körperteilen bedeutend, so in 1 qcm am grössten (328) an der Stirn und am geringsten (123) an der Streckseite des Vorderarms; also ist die relative Schwankungsbreite ca. $2.7: 1$ (Tab. I und II).

Das Volumen der angenommenen einzelnen Schweissdrüse ( $\frac{\text { Menge der Schweissdrüse in } 1 \mathrm{gcm}}{\text { Zahl der Ausmündung in } 1 \mathrm{gcm}}$ ) ist auch nach den Körperteilen verschieden gross, so an der Stirn am grössten $(0.038548 \mathrm{cmm})$ und am Unterschenkel, Vorderarm, Oberschenkel und Rücken überall fast gleich gross und an der Streckseite des Oberarms am kleinsten (0.021073 cmm); also die relative Schwankungsbreite ca. 1.8:1 (Tab. I und II).

Die genaueren gegenseitigen Verhāltnisse zwischen der Menge des Drüsenindividuums, der der Schweissdrüse und der Zahl der Ausmündung in einem bestimmten Hautfächeninhalt sind in Tabelle II angegeben; daraus kann man leicht ersehen, dass die Drüsenmenge und die Ausmūndungszahl in einem bestimmten Hautflächeninhalt an der Stirn und dem Hinterhaupt ganz miteinander parallel stehen, wäbrend an den übrigen Körperteilen sie mimimale Schwankungen je nach den Körperteilen aufweisen. Die relative Schwankungsbreite der Menge des Drüsenindividuums bei den verschiedenen Körperteilen $(1.8: 1)$ ist kleiner als die der Ausmündungszahl $(2.7: 1)$ und die der Drüsenmenge $(4: 1)$ in $1 \mathrm{qcm}$ Hautstūck. Deshalb kann man mit Sicherheit sagen, dass das Volumen des Drüsenindividuums nicht so beträchtliche individuelle Schwankung nach den Körperteilen als die der Drüsenmenge und die der Ausmündungszahl in 1 qcm Hautstūck zeigt.

Die Tabelle III zeigt, dass das durchschnittliche Volumen der 
Schweissdrüse unter den drei eingeteilten Hauptkörperteilen-Kopf, Rumpf und Extremitäten-im Kopf am grössten ist (9.7089 $\mathrm{cmm}$ in $1 \mathrm{qcm}$ ), dann folgt der Rumpf (4.3584 $\mathrm{cmm})$ und am geringsten ist es in den Extremitäten $(4.0821 \mathrm{cmm})$, so ist der relative Grenzwert ca. $2.5: 1$. Im Kopf ist die Drüsenmenge an der Stirn ca. 2 mal grösser als am Hinterhaupt, aber die Ausmündungszahl an jener ein wenig grösser als bei diesem; so ist das Volumen des Drüsenindividuums an der Stirn viel grösser $(0.038548 \mathrm{cmm})$ als am Hinterhaupt $(0.022431 \mathrm{cmm})$. Im Rumpf ist die Schweissdrüsenmenge am Rücken etwas grösser als am Bauch, während die Zahl der Ausmündung ganz umgekehrt sich verhält ; so ist das Volumen des Drüsenindividuums am Rücken grösser $(0.02997 \mathrm{cmm})$ als am Bauch $(0.025345 \mathrm{cmm})$. In den Extremitäten ist die Drüsenmenge an der unteren $(4.6622 \mathrm{cmm}$ in $1 \mathrm{qcm})$ viel grösser als an der oberen $(3.5021 \mathrm{cmm})$, und die obere Extremität besitzt am Vorderarm $(4.0118 \mathrm{cmm})$ eine viel grössere Drüsenmenge als am Oberarm $(2.9923 \mathrm{cmm})$ sowie die untere Extremität am Unterschenkel $(5.3292 \mathrm{cmm})$ als am Oberschenkel (3.9952 cinm). Also kann man sagen, dass in den Extremitäten die Menge der Schweissdrüsen an den distalen Regionen viel reichlicher als an den proximalen ist. In den Extremitäten sind die Zahl der Ausmündung und das Volumen des Drüsenindividuums an der unteren grösser als an der oberen, wie die Drüsenmenge in $1 \mathrm{qcm}$, und in der oberen Extremität ist die Zahl der Ausmündung am Oberarm (142) grösser als am Vorderarm (123), aber das Volumen des Drüsenindividuums an jenem kleiner als an diesem, in der unteren Extremität sind sie am Unterschenkel grösser als am Oberschenkel. Wie die Drüsenmenge in $1 \mathrm{qcm}$ sind die Zahl der Ausmündung und das Volumen des Drüsenindividuums in den Extremitäten an den distalen Regionen reichlicher als an den proximalen.

2. Das Volumen der Talgdrüsen auf $1 \mathrm{qcm}$ Hautflächeninhalt (Tab. IV) ist an der Stirn am grössten $(9.2911 \mathrm{cmm})$, damn folgen das Hinterhaupt $(9.2498 \mathrm{cmm})$, der Rücken $(0.4643 \mathrm{cmm})$ und die Streckseite des Vorderarms $(0.3599 \mathrm{cmm})$ u.s.w. und am geringsten ist es an der lateralen Seite des Unterschenkels $(0.0424 \mathrm{~mm})$ und diese in $1 \mathrm{qcm}$ Hautstück befindliche Drüsenmenge am Unterschenkel ist kleiner als die der durchschnittliche Menge der Talgdrüsen der nur einen Haargruppe am Hinterhaupt $(0.0701 \mathrm{cmm})$ und an der Stirn $(0.0652 \mathrm{cmm})$, so dass die relative Schwankungsbreite auffallend gross ist (ca. $220: 1$ ).

Die durchschnittliche Menge der Talgdrüsen der einen Haargruppe ist am Hinterhaupt $(0.070137 \mathrm{cmm})$ am grössten, dann folgen die Stirn $(0.065256 \mathrm{cmm})$, der Rücken $(0.018866 \mathrm{cmm})$, die Streckseite des Vorderarms $(0.016628 \mathrm{cmm})$ u.s.w., und ist die Lateralseite des Unter- 
schenkels am geringsten $(0.002358 \mathrm{cmm})$, so ist die relative Schwankungsbreite ca. $30: 1$.

In den 8 Körperteilen habe ich je 10 Haargruppen ganz frei absichtlos gewählt und verfolgt, um die Haargruppe herauszufinden, die mit der Talgdrüse keinen Zusammenhang hat, und konnte an 3 unter den 8 untersuchten Körperteilen solche Haargruppe beobachten, so je 1 unter 10 Haargruppen der Streckseite des Oberarms, der Lateralseite des Unterschenkels, je 2 an der Lateralseite des Oberschenkels (Tab. 18-25).

Die Haargruppen ohne Begleitung der Talgdrüse kommen häufig auf den Körperteilen der wenigen Talgdrüsenmenge in einem bestimmten Hautflächeninhalt vor.

In den 3 Hauptabteilungen des Körpers fand ich, dass die Menge der Talgdrüsen in $1 \mathrm{qcm}$ Hautflächeninhalt im Kopf am grössten ist (9.2705 $\mathrm{cmm})$, dann folgt der Rumpf $(0.3122 \mathrm{cmm})$, am geringsten ist sie in den Extremitäten $(0.1648 \mathrm{cmm})$, indem die relative Schwankungsbreite sich auf ca. $56: 1$ beläuft (Tab. V). Im Kopf ist die Menge an der Stirn ein wenig grösser als am Hinterhaupt. Im Rumpf ist sie am Rücken $(0.4643 \mathrm{cmm}$ in $1 \mathrm{gcm})$ grösser als am Bauch $(0.1601 \mathrm{cmm})$, also auf der Dorsalseite ca. 3 mal grösser als auf der Ventralseite. In den Extremitäten ist sie an der oberen ca. 3 mal grösser als an der unteren und an den distalen Regionen $-(0.2012 \mathrm{cmm})$ reichlicher als an den proximalen $(0.1285 \mathrm{cmm})$. In der oberen Extremität ist sie am Vorderarm reichlicher als am Oberarm. In der unteren Extremität ist sie am Oberschenkel reichlicher als am Unterschenkel.

Noch erkennt man aus der Tabelle V, dass die durchschnittliche Menge der Talgdrüsen der einen Haargruppe im Kopf am grössten ist $(0.067697 \mathrm{cmm})$, daun folgt der Rumpf $(0.012362 \mathrm{cmm})$, am kleinsten ist sie in den Extremitäten $(0.007712 \mathrm{cmm})$, indem die relative Schwankungsbreite sich auf ca. $9: 1$ beläuft. Im Kopf ist diese Menge am Hinterhaupt $(0.070138 \mathrm{cmm})$ etwas grösser als an der Stirn $(0.065256$ cmm). Im Rumpf ist sie am Rücken ca. 3 mal grösser als am Bauch. In den Extremitäten ist sie an der oberen reichlicher als an der unteren und bei distal $(0.009493 \mathrm{cmm})$ reichlicher als proximal $(0.005931 \mathrm{cmm})$. In der oberen Extremität ist die Menge am Vorderarm $(0.016628 \mathrm{cmm})$ reichlicher als am Oberarm $(0.007369 \mathrm{cmm})$. In der unteren Extremität ist sie am Oberschenkel 2 mal grösser als am Unterschenkel.

3. Die Menge der Haarbalgmuskeln in $1 \mathrm{qcm}$ Hautflächeninhalt (Tab. VI) ist am Hinterhaupt (3.1701 cmm) am grössten, dann folgen der Unterschenkel $(2.0963 \mathrm{cmm})$, die Stirn $(2.0275 \mathrm{cmm})$ und der Oberschenkel (1.9696 cmm) u.s.w., und sie ist an Bauch $(0.5359 \mathrm{cmm})$ 
arn geringsten, so dass die relative Schwankungsbreite ca. 6:1 beträgt.

Die durchschnittliche Menge der Muskeln der einen Haargruppe (Tab. VI) ist am Unterschenkel $(0.095655 \mathrm{cmm})$ am grössten, dann folgen der Oberschenkel $(0.086911 \mathrm{cmm})$, der Vorderarm $(0.060459 \mathrm{cmm})$ und der Oberarm (0.057056 cmm) u.s.w., und sie ist an der Stirn (0.015546 cunm) am geringsten, so dass die relative Schwankungsbreite ca. 6:1 beträgt.

Die relative Schwankungsbreite der Muskelmenge in $1 \mathrm{qcm}$ Hautflächeninhalt $(6: 1)$ ist zwar gleich der der durchschnittlichen Menge der Muskeln der einen Haargruppe $(6: 1)$, aber die im bestimmten Hautflächeninhalt vorkommende Muskelmenge geht nicht immer mit der durchschnittlichen Menge der Muskeln der einen Haargruppe Hand in Hand. Nämlich die Menge der Muskeln in einem bestimmten Hautflächeninhalt im Kopf ist viel grösser als in den Extremitäten, während die durchschnittliche Menge der Muskeln der einen Haargruppe im ersteren deutlich weniger als in den letzteren ist.

Die Mengenverhältnisse in den drei Hauptkörperteilen sind in der Tabelle (VII) angegeben, so ist die Menge des Haarbalgmuskels im Kopf $(2.5988 \mathrm{cmm}$ in $1 \mathrm{qcm})$ am grössten, dann folgen die Extremitäten $(1.7236 \mathrm{cmm})$ und im Rumpf $(0.8499 \mathrm{cmm})$ ist sie am geringsten, indem die relative Schwankungsbreite sich auf ca. 3:1 beläuft. Im Kopf ist die Menge am Hinterhaupt (3.1701 $\mathrm{cmm}$ ) viel grösser als an der Stirn (2.0275 cmm). Im Rumpf ist sie am Rücken $(1.1638 \mathrm{cmm})$ ca. 2 mal grösser als am Bauch $(0.5359 \mathrm{cmm})$. In den Extremitäten ist sie an der unteren reichlicher als an der oberen und bei distal $(1.7370 \mathrm{cmm})$ ein wenig reichlicher als proximal $(1.7101 \mathrm{cmm})$. In der oberen Extremität ist die Menge am Oberarm $(1.4506 \mathrm{cmm})$ reichlicher als am Vorderarm $(1.3777 \mathrm{cmm})$. In der unteren Extremität ist sie am Unterschenkel $(2.0963 \mathrm{cmm})$ ein wenig reichlicher als am Oberschenkel $(1.9696 \mathrm{cmm})$.

Die Tabelle VII zeigt die durchschnittliche Menge der Muskeln der einen Haargruppe. Aus dieser Tabelle kann man leicht ersehen, dass die Menge des einzelnen Muskels in den Extremitäten (0.075020 cmm) am grössten ist, dann folgt der Rumpf $(0.033345 \mathrm{cmm})$; am kleinsten ist sie im Kopf $(0.021105 \mathrm{cmm})$, indem die relative Schwankungsbreite sich auf ca. 4:1 beläuft. Im Kopf ist die einzelne Menge am Hinterhaupt $(0.026664 \mathrm{cmm})$ grösser als an der Stirn $(0.015546 \mathrm{cmm})$. Im Rumpf ist sie am Rücken $(0.047803 \mathrm{cmm})$ viel grösser als am Bauch $(0.018886$ cmm). In den Extremitäten ist sie an der unteren $(0.091283 \mathrm{cmm})$ viel grösser als an der oberen $(0.058758 \mathrm{cmm})$ und bei beiden distal $(0.078057$ 
cmm) ein wenig grösser als proximal $(0.071984 \mathrm{cmm})$. In der oberen Extremität ist die Menge am Vorderarm $(0.060459 \mathrm{cmm})$ reichlicher als am Oberarm $(0.057056 \mathrm{cmm})$. In der unteren Extremität ist sie am Unterschenkel $(0.095655 \mathrm{cmm})$ etwas grösser als am Oberschenkel (0.086911 cmm).

Wenn man nuu die Verhältnisse der Gesamtmenge der drei obigen Hautanhangsorgane (Muskel, Talg- und Schweissdrüse) in verschiedenen Körperregionen recht bedenkt, so erkennt man aus den Tabellen III, V und VII folgendes, nämlich die Gesamtmenge ist ‘im Kopf am grössten (21.5782 cmm in $1 \mathrm{qcm})$, dann folgen der Rumpf (5.9942 cmm) und die Extremitäten $(5.0968 \mathrm{cmm})$, während die relative Schwankungsbreite ca. $4: 1.2: 1$ zeigt. Im Kopf ist die gesamte Menge an der Stiru (23.9623 cmm) reichlicher als am Hinterhaupt (19.1940 cmm). Im Rumpf ist sie am Rücken $(6.2135 \mathrm{cmm})$ grösser als am Bauch (4.8273 $\mathrm{cmm}$ ). In den Extremitäten ist sie an der unteren $(6.9595 \mathrm{cmm}$ ) reichlicher als an der oberen $(4.9817 \mathrm{cmm})$, und die obere Extremität besitzt am Vorderarm $(5.7494 \mathrm{cmm})$ eine grössere Gesamtmenge als am Oberarm $(4.6115 \mathrm{cmm})$ sowie die untere Extremität mehr am Unterschenkel $(7.4679 \mathrm{cmm})$ als am Oberschenkel $(6.0531 \mathrm{cmm})$. Also kann man wohl sagen, dass in den Extremitäten die gesamte Menge der Hautanhangsorgane an den distalen Regionen $(6.6087 \mathrm{cmm})$ viel reichlicher als an den proximalen $(5.3223 \cdot \mathrm{cmm})$ ist.

Es wäre nun sehr interessant, die Befunde von verschiedenen Menchenrassen mit den obigen Ergebnissen zu vergleichen. Aber dieses Mal beschränkte ich mich bei der Beschreibung nur auf die Angabe eigener Befunde.

Zum Schluss spreche ich Herrn Professor K. Okajima für seine stete liebenswürdige Unterstützung bei dieser Arbeit meinen herzlichsten Dank aus. 